\title{
CLASSIFICATION OF MATERIAL G-STRUCTURES *
}

\author{
DAVID MARÍN MANUEL DE LEÓN
}

January 25, 2004

\begin{abstract}
A geometrical interpretation of the $G$-structures associated to elastic material bodies is given. In addition, characterizations of their integrability are obtained. Since the lack of integrability is a geometrical measure of the lack of homogeneity, the corresponding inhomogeneity conditions are obtained.
\end{abstract}

\section{Introduction}

The continuous theories of inhomogeneities were introduced by W. Noll [27]. In fact, Noll defined the notion of uniformity of a hyperelastic material body using only the constitutive law, which expresses the mechanical response of the elastic body in terms of the gradient of the deformation. Thus, a body is uniform if we can connect two arbitrary different points via a material isomorphism, that is, a linear isomorphism between the corresponding tangent spaces such that the mechanical response at both points is the same. The notion of material symmetry at a point also appears in a very natural way as a linear transformation of the tangent space at the point which does not change the mechanical response. These notions can be translated in a modern geometrical language in terms of Lie groupoids and Lie groups. Indeed, the uniformity permits to construct a $G$-structure on the body manifold whose integrability is equivalent to the local homogeneity of the material body.

The work by Noll was extended by C.C. Wang [34 in a setting of principal bundles, but without an explicit mention of the theory of $G$-structures (see also [4]). The first time that the theory of $G$-structures appears explicitly linked to uniformity occurs in a paper by

*(2000) MS Classification: 53C10, 74B20, 74E05. PACS numbers: 03.40.Dz, 02.40.Ma. Key words: Uniformity, homogeneity, $G$-structures, integrability, Lie groupoids, connections. 
Elzanowski, Epstein \& Sniatycki [9]. In that paper, the authors have also considered several types of $G$-structures corresponding to different kinds of materials. However, a systematic study of the integrability of the so-called material $G$-structures is not available up to our knowledge. This is just the aim of the present paper. For a material $G$-structure we mean a $G$-structure on a material body where $G$ is a Lie subgroup of the special general group $S l(3, \mathbb{R})$. We use a classification of these subgroups usually attributed to S. Lie [23, 28, 34].

The first remarkable fact is the difficulty to obtain integrability conditions for some of these $G$-structures in contrast with the low dimension that we are considering. The second point to remark is the additional difficulties arising from the fact that we are considering subgroups of $S l(3, \mathbb{R})$ instead of subgroups of $G l(3, \mathbb{R})$. All these difficulties are conveniently discussed along the paper.

The paper is structured as follows. In Section 2 we discuss $G$-structures defined by tensors in a general setting (the manifold is not necessarily three-dimensional). We give an slight generalization of some results contained in [1] for nonlinear "tensors". Moreover, we establish some properties concerning $G$-structures obtained by intersecting and enlarging. These results will be very useful later. The integrability of general $G$-structures is studied in Section 3. We propose a new method to do this, using local $G$-connections instead of global ones. The method leads us to integrability conditions involving linear partial differential equations whereas the usual procedures lead to more complicated PDE's. Section 4 is devoted to discuss some $G$-structures defined by tensors, in particular, vector fields, one-forms, two-forms, metrics and tensor fields of type $(1,1)$. We use the results previously obtained by E.T. Kobayashi [13, 14] and J. Lehmann-Lejeune [17] for 0-deformable tensor fields. We notice the amazing similarity between the definition of 0-deformability in [12] and the notion of uniformity. In Section 5 we recall the formulation of the continuous theories of inhomogeneities in geometrical terms. Thus, the uniformity of the body permits to associate with it a Lie groupoid, in such a way that, fixing a linear frame at a point (a reference crystal) one obtains a $G$-structure, where $G$ is the isotropy group at that point. Notice that this $G$-structure is defined modulo conjugation, but this is sufficient for our purposes, since the integrability is not affected by conjugation. In Section 6, after recalling the classification of the connected subgroups of $S l(3, \mathbb{R})$ modulo conjugation, we give a geometrical interpretation of the corresponding $G$-structures, and we simultaneously obtain in many cases the integrability condition. When the integrability condition is expressed in terms of the vanishing of some tensor fields, they would be just the inhomogeneity tensors for the corresponding material. Finally, in Section 7, we recall a classic theorem due to Chevalley and we give some applications. Using the natural representation, it implies that for each algebraic subgroup $G$ of $G l(n, \mathbb{R})$, every $\mathcal{N}(G)$-structure is given by the projectivization of a tensor field which is sum of 0 -deformable tensor fields, where $\mathcal{N}(G)$ is the normalizer of $G$ in $G l(n, \mathbb{R})$. 


\section{$2 G$-structures defined by tensors}

Along this paper, $\left\{e_{1}, \ldots, e_{n}\right\}$ will denote the canonical basis of $\mathbb{R}^{n}$, and $\left\{e^{1}, \ldots, e^{n}\right\}$ its dual basis. The space of tensors of type $(r, s)$ will be denoted by $T_{r}^{s} \mathbb{R}^{n}=\left(\mathbb{R}^{n}\right)^{\otimes r} \otimes\left(\left(\mathbb{R}^{n}\right)^{*}\right)^{\otimes s}$. We also notice that the action of $G l(n, \mathbb{R})$ over $\operatorname{End}\left(\mathbb{R}^{n}\right)=\mathbb{R}^{n} \otimes\left(\mathbb{R}^{n}\right)^{*}$ is the functorial action induced by the adjoint representation $A \mapsto\left(B \mapsto A B A^{-1}\right)$.

Let $M$ be an $n$-dimensional manifold and denote by $\mathcal{F} M$ its linear frame bundle. $\mathcal{F} M$ is a principal bundle over $M$ with projection $\pi: \mathcal{F} M \longrightarrow M$ and structure group $G l(n, \mathbb{R})$. A $G$-structure $P$ on $M$ is just a $G$-reduction of $\mathcal{F} M$ (see [1, 6, 8, 11, 16]).

Assume that $G l(n, \mathbb{R})$ acts on a manifold $F$ on the left. Fixing an element $u \in F$ we denote by $G_{u}$ the isotropy group at $u$, and by $F_{u}$ the orbit of the action through $u$. Thus, we have

$$
\begin{aligned}
G_{u} & =\{a \in G l(n, \mathbb{R}) \mid a u=u\}, \\
F_{u} & =\{a u \mid a \in G l(n, \mathbb{R})\} .
\end{aligned}
$$

Definition 2.1 An F-tensor on $\mathcal{F} M$ is a differentiable mapping $t: \mathcal{F} M \longrightarrow F$ such that $t(z a)=a^{-1} t(z)$, for all $a \in G l(n, \mathbb{R})$ and $z \in \mathcal{F} M$.

The following result gives the family of $G$-structures defined by tensors. It is a slight generalization of that proved in [1].

Theorem 2.2 Giving a $G_{u}$-structure on $M$ is the same as giving an $F$-tensor on $\mathcal{F} M$ which satisfy the following two conditions:

(i) $t$ takes values in $F_{u}$;

(ii) $t$ is a differentiable map of $\mathcal{F} M$ into $F_{u}$.

The proof is omitted, since it is a direct translation of that in [11. We only remark that the relation between $t$ and the $G_{u}$-structure is given by the formula $P_{u}=t^{-1}(u)$.

Remark 2.3 In the case where $F_{u}$ is an embedded submanifold of $F$, then an $F$-tensor $t$ which take values in $F_{u}$ is automatically differentiable as a map $t: \mathcal{F} M \longrightarrow F_{u}$. This is the case if $F_{u}$ is locally compact. For instance, if $G$ is a real algebraic subgroup of $G l(n, \mathbb{R})$ (see [1]).

Remark 2.4 If $u_{1}$ and $u_{2}$ are in the same orbit, say $u_{1}=a u_{2}$, for some $a \in G l(n, \mathbb{R})$, then $G_{u_{1}}=a G_{u_{2}} a^{-1}$ and $P_{u_{1}}=P_{u_{2}} a$, that is, $P_{u_{1}}$ and $P_{u_{2}}$ are conjugate. 
Since $G l(n, \mathbb{R})$ acts on $F$ we can construct the associated bundle $E=(\mathcal{F} M \times F) / G l(n, \mathbb{R})$ over $M$ with typical fiber $F$. Let us recall that $E$ consists of the equivalence classes of pairs $(p, \xi) \in \mathcal{F} M \times F$ such that $(p, \xi) \sim\left(p a, a^{-1} \xi\right)$.

Proposition 2.5 (see [11] for the linear case). There exists a one-to-one correspondence between F-tensors and sections of $E$.

Proof: In fact, given an $F$-tensor $t$ we define $\sigma_{t}: M \longrightarrow E$ by $\sigma_{t}(x)=[(p, t(p)]$, where $p$ is a linear frame at $x$.

Remark 2.6 The above correspondence is nothing but the extension of the classical definition of tensor fields. Given a basis $p$ of the tangent space $T_{x} M$ we associate the components $(t(p))$ to the tensor $t$, which change according to the well-known rule. It should be remarked that, if $p \in P$, where $P$ is the $G_{u}$-structure defined by $t$, then $\sigma_{t}(x)=[(p, u)]$ (see [15]).

Corollary 2.7 Assume that $H$ is a closed subgroup of $G l(n, \mathbb{R})$. Then there exists a one-toone correspondence between $H$-structures and sections of the principal bundle $\mathcal{F} M / H$.

Proof: Take $F=G l(n, \mathbb{R}) / H$ and $u=[e]$, where $e$ denotes the neutral element of $G l(n, \mathbb{R})$. Thus, $G l(n, \mathbb{R})$ acts on the homogeneous space $F$ in the obvious manner, and we have $F_{u}=F$. Since $\mathcal{F} M / H \cong(\mathcal{F} M \times G l(n, \mathbb{R}) / H) / G l(n, \mathbb{R})$ we deduce the result.

Remark 2.8 It should be noticed that Theorem [2.2, Proposition 2.5] and Corollary 2.7] are still true for arbitrary principal bundles, with the obvious extension of the notion of $F$-tensor. In this way, every $H$-reduction of the structure group $G$ of a principal bundle $P$ to a closed subgroup $H$ may be viewed as defined by an $G / H$-tensor on $P$.

Let $F$ and $F^{\prime}$ be two manifolds on which $G l(n, \mathbb{R})$ acts on the left, and $\phi: F \longrightarrow F^{\prime}$ a $G l(n, \mathbb{R})$-invariant differentiable mapping, i.e., $\phi(a \xi)=a \phi(\xi)$, for all $x \in F, a \in G l(n, \mathbb{R})$. Given a point $u \in F$ we denote by $G_{u}$ and $G_{u^{\prime}}^{\prime}$ the isotropy groups of $u$ and $u^{\prime}=\phi(u)$, respectively. It si easy to check that $\phi$ induces a mapping between the associated fiber bundles, namely $\Phi: E=(\mathcal{F} M \times F) / G l(n, \mathbb{R}) \longrightarrow E^{\prime}=\left(\mathcal{F} M \times F^{\prime}\right) / G l(n, \mathbb{R})$ given by $\Phi([p, a])=[p, \phi(a)]$. Thus, given a section $\sigma$ of $E$ we obtain a section $\Phi \circ \sigma$ of $E^{\prime}$. Therefore, we have obtained a way to relate $G_{u}$ and $G_{u^{\prime}}^{\prime}$-structures. If $P$ is a $G_{u^{-}}$structure defined by an $F$-tensor $t$, we obtain a $G_{u^{\prime}}^{\prime}$-structure defined by an $F^{\prime}$-tensor $t^{\prime}$ according to Proposition 2.5. In fact, $t$ induces a section $\sigma_{t}$ of $E$ and $t^{\prime}$ is given by the section $\Phi \circ \sigma_{t}$. Notice that $G_{u} \subset G_{u^{\prime}}^{\prime}$. The above procedure corresponds to enlarge the structure group. Conversely, given a $G_{u^{\prime}}^{\prime}$-structure, we can detect its reducibility to a $G_{u^{-}}$-structure by checking if the section $\sigma_{t^{\prime}}$ factorizes through $E$. 
Example 2.9 Consider the natural action of $G l(n, \mathbb{R})$ on $\mathbb{R}^{n}$ and denote by $S_{k}\left(\mathbb{R}^{n}\right)$ the Stiefel manifold of $k$ frames of $\mathbb{R}^{n}$. By $G_{k}\left(\mathbb{R}^{n}\right)$ we will denote the Grassmannian of $k$ planes in $\mathbb{R}^{n}$. There exists a canonical mapping $\phi: S_{k}\left(\mathbb{R}^{n}\right) \longrightarrow G_{k}\left(\mathbb{R}^{n}\right)$ which assigns to each $k$ frame $u$ the $k$-plane $u^{\prime}=\langle u\rangle$ generated by it. Let $u$ be the $k$ frame consisting of the $k$ first elements of the standard basis of $\mathbb{R}^{n}$. A direct computation shows that

$$
G_{u}=\left\{\left(\begin{array}{cc}
I_{k} & B \\
0 & C
\end{array}\right) \mid A \in G l(k, \mathbb{R}), C \in G l(n-k, \mathbb{R})\right\}
$$

where $I_{k}$ denotes the identity matrix of order $k$. Moreover, we get

$$
G_{u^{\prime}}^{\prime}=\left\{\left(\begin{array}{cc}
A & B \\
0 & C
\end{array}\right) \mid A \in G l(k, \mathbb{R}), C \in G l(n-k, \mathbb{R})\right\} .
$$

Alternatively, we can describe $G_{u^{\prime}}^{\prime}$ as follows:

$$
G_{u^{\prime}}^{\prime}=\{a \in G l(n, \mathbb{R}) \mid \exists \Lambda \in G l(k, \mathbb{R}), a u=\Lambda u\}
$$

For $k=1$, we have that $G_{1}\left(\mathbb{R}^{n}\right)$ is the projective space $\mathbb{P R}^{n}$ and $G_{u^{\prime}}^{\prime}=\{a \in G l(n, \mathbb{R}) \mid \exists \lambda \in$ $\left.\mathbb{R}^{*}, a u=\lambda u\right\}=\left\{\lambda a \mid \lambda \in \mathbb{R}^{*}, a \in G \operatorname{Ln}\right\}=\mathbb{R}^{*} G_{u}$.

It should be noticed that the action of $G l(n, \mathbb{R})$ is transitive and hence the orbits of $u$ and $u^{\prime}$ are the whole manifolds $S_{k}\left(\mathbb{R}^{n}\right)$ and $G_{k}\left(\mathbb{R}^{n}\right)$, respectively.

To end this example, take a section $\sigma_{t^{\prime}}$ of $E^{\prime}=\left(\mathcal{F} M \times G_{k}\left(\mathbb{R}^{n}\right)\right) / G l(n, \mathbb{R})$ associated with an $F_{u^{\prime}}^{\prime}$-tensor $t^{\prime} . \sigma_{t^{\prime}}$ maps each point $x \in M$ into a $k$-plane in $T_{x} M$, or, in other words, a $k$-dimensional distribution on $M$. Conversely, given a $k$-dimensional distribution on $M$, we can construct the corresponding $F_{u^{\prime}}^{\prime}$-tensor.

Next, consider two $G$-structures $P_{1}$ and $P_{2}$ defined by an $F_{1}$-tensor $t_{1}$ and an $F_{2}$-tensor $t_{2}$, respectively. We assume that there is a section of $\mathcal{F} M$ which takes values into $P_{1} \cap P_{2}$. Here $F_{1}$ and $F_{2}$ are manifolds on which $G l(n, \mathbb{R})$ acts on the left. We assume that $P_{1}=t^{-1}\left(u_{1}\right)$ and $P_{2}=t^{-1}\left(u_{2}\right)$, where $u_{1} \in F_{1}$ and $u_{2} \in F_{2}$. The corresponding structure groups are the isotropy groups $G_{u_{1}}$ and $G_{u_{2}}$. Define an action of $G l(n, \mathbb{R})$ on the product manifold $F=F_{1} \times F_{2}$ in the natural way, namely $a\left(\xi_{1}, \xi_{2}\right)=\left(a \xi_{1}, a \xi_{2}\right)$. Fixing a point $u=\left(u_{1}, u_{2}\right) \in F$, we deduce that

$$
G_{u}=G_{u_{1}} \cap G_{u_{2}}, F_{u} \subset F_{u_{1}} \times F_{u_{2}} .
$$

Define now an $F$-tensor $t$ on $\mathcal{F} M$ by

$$
t(p)=\left(t_{1}(p), t_{2}(p)\right.
$$


A direct computation shows that $t$ takes values in $F_{u}$. We assume that $t$ is smooth as a mapping from $\mathcal{F} M$ into $F_{u}$ (this happens if $F_{u}$ is an embedded submanifold of $F_{1} \times F_{2}$, for instance). Moreover, we have $t^{-1}(u)=t_{1}^{-1}\left(u_{1}\right) \cap t_{2}^{-1}\left(u_{2}\right)$, from which we deduce that $t$ defines a $\left(G_{u_{1}} \cap G_{u_{2}}\right)$-structure on $M$.

Conversely, given an $F$-tensor $t$, we can recover $t_{1}$ and $t_{2}$ by composing $t$ with the canonical projections $F \longrightarrow F_{1}$ and $F \longrightarrow F_{2}$. Thus, we have proved the following.

Proposition 2.10 The intersection of two G-structures defined by tensors is a new G-structure defined by a tensor and with structure group the intersection of both groups.

Finally, a direct application of Theorem 2.2 for arbitrary principal bundles (see Remark 2.8) yields the following construction.

Let $G$ and $G_{1}$ be closed subgroups of $G l(n, \mathbb{R})$ such that $G \subset G_{1} \subset G l(n, \mathbb{R})$, and assume that $G_{1}$ acts on a manifold $F$, and $G$ is the isotropy group of $u \in F$ under this action. Notice that we suppose that only $G_{1}$ acts on $F$, not necessarily the whole group $G l(n, \mathbb{R})$.

Proposition 2.11 Giving a $G$-structure on $M$ is the same as giving a $G_{1}$-structure $P_{1}$ and an F-tensor $t$ on $P_{1}$ such that

(i) $t$ takes values in $F_{u}$.

(ii) $t$ is a differentiable map of $P_{1}$ into $F_{u}$.

Of course, Proposition 2.11 can be applied to the situation of a $G_{1}$-structure and a $G_{2^{-}}$ structure defined by two tensors, by considering $G_{1} \cap G_{2} \subset G_{1} \subset G l(n, \mathbb{R})$, and the action of $G_{1}$ on $F=F_{1} \times F_{2}$. Thus, the $\left(G_{1} \cap G_{2}\right)$-structure is obtained by reducing first $\mathcal{F} M$ to $G_{1}$, and, then, defining an $F$-tensor on the $G_{1}$-reduction $P_{1}$.

Example 2.12 Consider the Grassmannian manifold $F_{1}=G_{k}\left(\mathbb{R}^{n}\right)$, and the natural action of $G l(n, \mathbb{R})$ on it. Let $u_{1}$ be the $k$-plane spanned by the first $k$ elements of the standard basis of $\mathbb{R}^{k}$. Thus, $G_{u_{1}}$ is given by (1). As we know, a $G_{u_{1}}$-structure is just a $k$-dimensional distribution $D$ on $M$. Let $F$ be the vector space of positive definite symmetric covariant tensors of order 2 on $\mathbb{R}^{k}$. $G_{u_{1}}$ acts on $\mathbb{R}^{k}$, but this is not the case for $G l(n, \mathbb{R})$ ! Take an inner product $u$ on $\mathbb{R}^{k}$, say $u \in F$. The isotropy group $G$ of $u$ is just

$$
G=\left\{\left(\begin{array}{cc}
A & B \\
0 & C
\end{array}\right) \mid A \in O(k), C \in G l(n-k, \mathbb{R})\right\}
$$


Therefore, a $G$-structure on $M$ consists in a $k$-dimensional distribution $D$ on $M$ endowed with an inner product on each subspace $D_{x}, x \in M$. In other words, if we view a distribution on a manifold as a vector subbundle of the tangent bundle, a $G$-structure on $M$ consists of a vector subbundle with a fiber metric.

The above construction can be extended to include more general structures:

- Tangent $H$-structure on a $k$-distribution.

Assume that $G$ is the group

$$
G=\left\{\left(\begin{array}{cc}
A & B \\
0 & C
\end{array}\right) \mid A \in H \subset G L(k, \mathbb{R}), C \in G l(n-k, \mathbb{R})\right\} .
$$

Thus, giving a $G$-structure is equivalent to giving a $k$-dimensional distribution on $M$, and a "H-structure" on each vector subspace $D_{x}, x \in M$. This means that if $D$ is involutive then we have a $H$-structure on each leaf of the induced foliation.

- Transverse $H$-structure to a $k$-distribution.

On the other hand, assume that

$$
G=\left\{\left(\begin{array}{cc}
A & B \\
0 & C
\end{array}\right) \mid A \in G L(k, \mathbb{R}), C \in H \subset G l(n-k, \mathbb{R})\right\}
$$

Now, giving a $G$-structure is equivalent to giving a $k$-dimensional distribution on $M$, and a "H-structure" on each quotient vector space $T_{x} M / D_{x}$, for all $x \in M$. This means that if $D$ is involutive then we have a foliation with transverse $H$-structure. In such a case, we say that the $G$-structure is projectable if there exists a local reference $\left\{X_{1}, \ldots, X_{n}\right\}$ on an open subset $U$ in $M$ such that

$$
\left[X_{i}, X_{a}\right]=0,1 \leq i \leq k, 1 \leq a \leq n
$$

This implies that the local quotient manifold $U^{\prime} / D$ admits a $H$-structure, where $U^{\prime}$ is possible smaller than $U$.

We end this section with two examples of tangent and transverse $H$-structures.

Example 2.13 Let $H$ be the subgroup of $G l(2, \mathbb{R})$ given by

$$
H=\left\{\left(\begin{array}{ll}
a & 0 \\
0 & c
\end{array}\right) \mid a^{\beta}=c^{\alpha}\right\}
$$


with $\alpha, \beta \in \mathbb{N}$. A direct inspection shows that $H$ is the isotropy group of the tensor

$$
\underbrace{e^{1} \otimes \cdots \otimes e^{1}}_{\beta} \otimes \underbrace{e_{2} \otimes \cdots \otimes e_{2}}_{\alpha} .
$$

1. A tangent $H$-structure on a 2-dimensional distribution on a 3 -dimensional manifold $M$ is a $G$-structure with

$$
G=\left\{\left(\begin{array}{lll}
a & b & e \\
0 & d & f \\
0 & 0 & g
\end{array}\right) \in G l(3, \mathbb{R}), a^{\beta}=d^{\alpha}\right\},
$$

and it is given by a 2-dimensional distribution $D$ and a tangent tensor field $T$ of type $(\alpha, \beta)$, i.e., a section of $\xi^{\alpha, \beta}=\xi^{\otimes \alpha} \otimes\left(\xi^{*}\right)^{\otimes \beta}$ where $\xi$ is the vector sub-bundle $D \rightarrow M$ of $T M \rightarrow M$.

2. A transverse $H$-structure to a 1-dimensional distribution on a 3-dimensional manifold $M$ is a $G$-structure with

$$
G=\left\{\left(\begin{array}{lll}
e & f & g \\
0 & a & b \\
0 & 0 & d
\end{array}\right) \in G l(3, \mathbb{R}), a^{\beta}=d^{\alpha}\right\}
$$

and it is given by a 1-dimensional distribution $L$ and a transverse tensor field $T$ of type $(\alpha, \beta)$, i.e., a section of $\xi^{\alpha, \beta}=\xi^{\otimes \alpha} \otimes\left(\xi^{*}\right)^{\otimes \beta}$ where $\xi$ is the quotient vector bundle $T M / L \rightarrow M$ of $T M \rightarrow M$.

\section{$3 \quad$ Integrability}

A $G$-structure $P$ on $M$ is said to be integrable if it is locally equivalent to the flat standard $G$-structure $\mathbb{R}^{n} \times G \rightarrow \mathbb{R}^{n}$, where $\operatorname{dim} M=n$ (see [1]). This is equivalent to the existence of local coordinates $\left(x^{i}\right)$ such that the local section $\left(x^{i}\right) \mapsto\left(x^{i}, \frac{\partial}{\partial x^{i}}\right)$ is adapted.

The main problem in the theory $G$-structures is to give geometric characterizations of their integrability. For this purpose, it is very useful the notion of a $G$-connection.

Definition 3.1 A linear connection $\nabla$ in $M$ is said to be a G-connection for a G-structure $P$ on $M$ if the parallel transport maps adapted frames into adapted frames.

Remark 3.2 Thus, a linear connection $\nabla$ on $M$ is a $G$-connection if its horizontal distribution is tangent to the reduced sub-bundle, or equivalently, $\nabla$ reduces to a connection on $P$. If the $G$-structure $P$ is defined by a tensor $t$ then $\nabla$ is a $G$-connection if and only if $\nabla K=0$, where $K$ is the tensor field on $M$ defined from $t$. 
Since the integrability problem is a local notion, we can consider only local $G$-connections.

Proposition 3.3 A G-structure $P$ is integrable if and only if around each point in $M$ there are an open neighbourhood $U$ and a locally flat $G$-connection $\nabla$ on $U$ (i.e., $\nabla$ is torsion less and with zero curvature).

\section{Proof:}

$[\Rightarrow]$ Let $\left(x^{i}\right)$ be local coordinates such that $\left(\frac{\partial}{\partial x^{i}}\right)$ is an adapted local frame of $P$. Then the connection $\nabla$ defined by

$$
\nabla_{\frac{\partial}{\partial x^{2}}} \frac{\partial}{\partial x^{j}}=0
$$

is locally flat.

$[\Leftarrow]$ Since the curvature of $\nabla$ is zero, the horizontal distribution defined by $\nabla$ on $\mathcal{F} M$ is involutive. Let $z(p)=\left(X_{1}(p), \ldots, X_{n}(p)\right)$ be an adapted frame of $P$ at $p \in M$. The leaf trough $z(p)$ of the foliation defined by $\nabla$ is totally contained in $P$ because of $\nabla$ is a $G$-connection. Therefore, this leaf defines a smooth parallel local section $\left(X_{1}, \ldots, X_{n}\right)$ of $P$ over a neighbourhood of $p$. Since $\nabla$ is torsionless and $\nabla_{X_{i}} X_{j}=0$ we obtain that $\left[X_{i}, X_{j}\right]=0$, i.e., there are coordinates $x^{1}, \ldots, x^{n}$ such that $\left\{X_{i}=\frac{\partial}{\partial x^{i}}\right\}$ is an adapted local frame of $P$.

Remark 3.4 If $\nabla$ depends smoothly on some parameters then the local frame $\left\{X_{1}, \ldots, X_{n}\right\}$ depends also smoothly on them.

We take an adapted frame $X_{1}, \ldots, X_{n}$ on a coordinate neighborhood $U$ and define an auxiliary linear connection $\widetilde{\nabla}$ on $U$ by means of

$$
\widetilde{\nabla}_{X_{i}} X_{j}=0, \quad \forall i, j=1, \ldots, n
$$

In other words, $\widetilde{\nabla}$ is the linear connection defined by the local parallelism $\left\{X_{1}, \ldots, X_{n}\right\}$. It is clear that $\widetilde{\nabla}$ is a $G$-connection (adapted to $P$ ). Moreover, any linear connection on $U$ is of the form

$$
\nabla=\widetilde{\nabla}+\tau
$$

where $\tau$ is a tensor field of type $(1,2)$ on $U$. If we put $\tau\left(X_{i}, X_{j}\right)=\tau_{i j}^{k} X_{k}$, for each $i=1, \ldots, n$ we can define the maps $\tau_{i}: U \rightarrow \mathfrak{g l}(n, \mathbb{R})$ by putting

$$
\tau_{i}(x)=\left(\tau_{i j}^{k}(x)\right) .
$$


Proposition 3.5 With the above notations, $\nabla$ is a $G$-connection if and only if for all $i=$ $1, \ldots, n$ the maps $\tau_{i}$ take values in the Lie algebra $\mathfrak{g}$ of $G$.

\section{Proof:}

$[\Rightarrow]$ Let $\mathcal{T}_{i}^{t}$ be the parallel transport operator with respect to $\nabla$ along the integral curves of $X_{i}$, then

$$
\tau_{i j}^{k} X_{k}=\nabla_{X_{i}} X_{j}=\frac{d}{d s} \mathcal{T}_{i}^{-s}\left(X_{j}(s)\right)
$$

If $\nabla$ is $G$-connection then $\mathcal{T}_{i}^{-s}\left(X_{j}(s)\right)=a_{i j}^{k}(s) X_{k}$, where the matrix $a_{i}(s)=\left(a_{i j}^{k}(s)\right)$ belongs to $G$. Therefore $\tau_{i j}^{k} X_{k}=\left(\frac{d}{d s} a_{i j}^{k}(s)\right) X_{k}$, and then $\tau_{i j}^{k} X_{k}=\left(\frac{d}{d s} a_{i j}^{k}(s)\right) X_{k} \in \mathfrak{g}$.

$[\Leftarrow]$ Let $c(s)$ be a curve on $U$ and $\dot{c}(s)=c^{\ell}(s) X_{\ell}(s)$ its tangent vector, where $X_{\ell}(s)=$ $X_{\ell}(c(s))$. Let $Y_{\ell}(s)$ be the parallel transport along $c$ of the frame $\left\{X_{1}(0), \ldots, X_{n}(0)\right\}$. Put $Y_{i}(s)=a_{i}^{j}(s) X_{j}(s)$. We will show that $A(s)=\left(a_{i}^{j}(s)\right)$ belongs to $G$. Indeed, we have

$$
\begin{aligned}
0 & =\nabla_{\dot{c}(s)} Y_{i}(s)=c^{\ell}(s) \nabla_{X_{\ell}(s)} a_{i}^{j}(s) X_{j}(s) \\
& =c^{\ell}(s)\left(a_{i}^{j}(s) t_{\ell j}^{k}(s) X_{k}(s)+X_{\ell}\left(a_{i}^{j}(s)\right) X_{j}(s)\right) \\
& =\left(c^{\ell}(s) a_{i}^{j}(s) t_{\ell j}^{k}(s)+c^{\ell}(s) X_{\ell}\left(a_{i}^{k}(s)\right)\right) X_{k}(s),
\end{aligned}
$$

Thus, we obtain the following identity:

$$
c^{\ell}(s)\left(A(s) t_{\ell}(s)\right)+\frac{d A}{d s}=0,
$$

where $t_{\ell}(s)$ is the matrix with entries $t_{\ell i}^{k}(s)$. Therefore we get

$$
A(s)=\exp \left(-\int_{0}^{s} c^{\ell}(u) t_{\ell}(u) d u\right) .
$$

We notice that the assumption $t_{\ell}(u) \in \mathfrak{g}$ implies $\int_{0}^{s} c^{\ell}(u) t_{\ell}(u) d u \in \mathfrak{g}$, thus, $A(s) \in G$. Indeed, $A(s)$ belongs to connected component of the identity of $G$.

According to Proposition 3.5 if $\nabla=\widetilde{\nabla}+\tau$ is a $G$-connection then we can think the tensor field $\tau$ as a map $\tau: U \rightarrow \operatorname{Hom}\left(\mathbb{R}^{\mathrm{n}}, \mathfrak{g}\right)$. Using this notation, the following result will be useful in order to characterize those $G$-connections $\nabla$ which are torsion free.

Proposition 3.6 The torsion tensor of the G-connection $\nabla=\widetilde{\nabla}+\tau$ is given by $T^{\nabla}=$ $T^{\tilde{\nabla}}+\partial_{\mathfrak{g}} \tau$, where $\partial_{\mathfrak{g}}: \operatorname{Hom}\left(\mathbb{R}^{\mathrm{n}}, \mathfrak{g}\right) \rightarrow \operatorname{Hom}\left(\bigwedge^{2} \mathbb{R}^{\mathrm{n}}, \mathbb{R}^{\mathrm{n}}\right)$ is the map defined by

$$
\left(\partial_{\mathfrak{g}} \tau\right)(x \wedge y)=\tau(x)(y)-\tau(y)(x) .
$$


It should be noticed that $\partial_{\mathfrak{g}}$ is just the operator defining the Spencer cohomology of the Lie algebra $\mathfrak{g}$ (see [11]). In fact, after the identification of $T_{x} M$ with $\mathbb{R}^{n}$ using the basis $X_{1}(x), \ldots, X_{n}(x)$, we obtain

$$
\partial_{\mathfrak{g}} \tau_{i j}^{k}(x)=\tau_{i j}^{k}(x)-\tau_{j i}^{k}(x) .
$$

Thus, the equivalence class of $T^{\tilde{\nabla}}$ in $\operatorname{Hom}\left(\bigwedge^{2} \mathbb{R}^{\mathrm{n}}, \mathbb{R}^{\mathrm{n}}\right) / \operatorname{Im} \partial_{\mathfrak{g}}$, is just the first structure tensor of the $G$-structure and the kernel of $\partial_{\mathfrak{g}}$ is the first prolongation $\mathfrak{g}^{(1)}$ of the Lie algebra $\mathfrak{g}$.

As a consequence of Proposition 3.6 we deduce that if $T^{\tilde{\nabla}}$ does not take values in $\operatorname{Im} \partial_{\mathfrak{g}}$ then the $G$-structure $P$ is not integrable. Indeed, if $\nabla$ would be a free torsion $G$-connection then

$$
T^{\tilde{\nabla}}\left(X_{i}, X_{j}\right)=-\left[X_{i}, X_{j}\right]=-\gamma_{i j}^{k} X_{k}=-\left(\partial_{\mathfrak{g}} \tau\right)_{i j}^{k} X_{k}
$$

\section{Remark 3.7}

(i) If $\partial_{\mathfrak{g}}$ is surjective then the structure tensor is not an obstruction for the integrability.

(ii) If $\partial_{\mathfrak{g}}$ is injective, then there is at most one tensor $\tau$ such that $\nabla$ is a free torsion $G$-connection. Moreover, any $G$-connection is determined by its torsion tensor.

(iii) If $\partial_{\mathfrak{g}}$ is bijective then there is a unique (global) free torsion $G$-connection.

The next issue is to investigate if, in addition to the torsionless condition, we can choose $\tau$ in such a way that the curvature $R^{\nabla}$ of $\nabla$ vanishes identically. In order to do this, we try to modify $\nabla$ by adding a new tensor field $S: U \rightarrow \operatorname{Hom}\left(\mathbb{R}^{\mathrm{n}}, \mathfrak{g}\right)$ such that $\bar{\nabla}=\nabla+S$ verifies both conditions. Concerning the first one, we have that

$$
T^{\bar{\nabla}}=0 \Longleftrightarrow \partial_{\mathfrak{g}} S=0 \Longleftrightarrow S \in \operatorname{ker} \partial_{\mathfrak{g}}=\mathfrak{g}^{(1)} .
$$

On the other hand, recalling that $\bar{\nabla}=\nabla+S=\widetilde{\nabla}+\tau+S$ and putting $\Delta=\tau+S$, we obtain the following result:

Proposition 3.8 The curvature of $\bar{\nabla}=\widetilde{\nabla}+\Delta$ vanishes if and only if

$$
X_{i}\left(\Delta_{j}\right)-X_{j}\left(\Delta_{i}\right)+\left[\Delta_{i}, \Delta_{j}\right]-\gamma_{i j}^{k} \Delta_{k}=0, \quad 1 \leq i<j \leq n
$$

where the functions $\gamma_{i j}^{k}$ are defined by $\left[X_{i}, X_{j}\right]=\gamma_{i j}^{k} X_{k}$.

Proof: Using that $\bar{\nabla}_{X_{i}} X_{j}=\Delta_{i j}^{k} X_{k}$ we obtain

$$
\begin{aligned}
& \bar{\nabla}_{X_{i}} \bar{\nabla}_{X_{j}} X_{k}=\Delta_{j k}^{\ell} \bar{\nabla}_{X_{i}} X_{\ell}+X_{i}\left(\Delta_{j k}^{m}\right) X_{m}=\Delta_{j k}^{\ell} \Delta_{i \ell}^{m} X_{m}+X_{i}\left(\Delta_{j k}^{m}\right) X_{m} \\
& \bar{\nabla}_{X_{j}} \bar{\nabla}_{X_{i}} X_{k}=\Delta_{i k}^{\ell} \bar{\nabla}_{X_{j}} X_{\ell}+X_{j}\left(\Delta_{i k}^{m}\right) X_{m}=\Delta_{i k}^{\ell} \Delta_{j \ell}^{m} X_{m}+X_{j}\left(\Delta_{i k}^{m}\right) X_{m} \\
& \bar{\nabla}_{\left[X_{i}, X_{j}\right]} X_{k}=\bar{\nabla}_{\gamma_{i j}^{\ell} X_{\ell}} X_{k}=\gamma_{i j}^{\ell} \bar{\nabla}_{X_{\ell}} X_{k}=\gamma_{i j}^{\ell} \Delta_{\ell k}^{m} X_{m} \text {, }
\end{aligned}
$$


so that

$$
R^{\bar{\nabla}}\left(X_{i}, X_{j}\right)\left(X_{k}\right)=\left(X_{i}\left(\Delta_{j k}^{m}\right)-X_{j}\left(\Delta_{i k}^{m}\right)+\Delta_{j k}^{\ell} \Delta_{i \ell}^{m}-\Delta_{i k}^{\ell} \Delta_{j \ell}^{m}-\gamma_{i j}^{\ell} \Delta_{\ell k}^{m}\right) X_{m}
$$

Therefore, using the matrix convention introduced in (3) we can write the condition $R^{\bar{\nabla}}=0$ as the system (4).

Remark 3.9 It should be noticed that the above conditions involve linear partial differential equations whereas the usual procedures lead to more complicated PDE's.

As an application of this method we will give a characterization of the integrability of the tangent $G$-structures.

Assume $G$ is the group

$$
G=\left\{\left(\begin{array}{cc}
A & B \\
0 & C
\end{array}\right) \mid A \in H \subset G L(k, \mathbb{R}), C \in G l(n-k, \mathbb{R})\right\}
$$

and let $P$ be a $G$-structure. As we have seen in section 2, $P$ is the reduction of a bigger geometrical structure which consists uniquely in a $k$-dimensional distribution $D$. If $P$ is integrable then $D$ is involutive and it defines a foliation. Furthermore, on each leaf $S_{c}$ we have an $H$-structure which is also integrable.

Conversely, assume that the distribution $D$ is involutive and that the induced $H$-structure on each leaf $S_{c}$ is integrable (here $c$ denotes a transverse coordinate). In this case, taking a local adapted frame $X_{1}, \ldots, X_{n}$ we can find a map

$$
\tau_{c}: S_{c} \rightarrow \operatorname{Hom}\left(\mathbb{R}^{\mathrm{k}}, \mathfrak{h}\right)
$$

such that the connection $\nabla$ defined on $S_{c}$ by

$$
\nabla_{X_{i}} X_{j}=\left(\tau_{c}\right)_{i j}^{\ell} X_{\ell}, \quad i, j=1, \ldots, k
$$

is a locally flat $H$-connection.

Proposition 3.10 If in addition $\tau_{c}$ depends smoothly on $c$, then $P$ is integrable.

Proof: Consider the inclusion $\rho: \mathfrak{h} \hookrightarrow \mathfrak{g}$. We define a smooth map

$$
\tau: U \rightarrow \operatorname{Hom}\left(\mathbb{R}^{\mathrm{n}}, \mathfrak{g}\right)
$$

by putting

$$
\tau(c, y)_{i j}^{\ell}= \begin{cases}\rho\left(\tau_{c}(y)\right)_{i j}^{\ell} & \text { if } i \leq k \\ 0 & \text { if } i>k\end{cases}
$$


with $j, \ell=1, \ldots, n$. The connection $\nabla$ defined by $\tau$ is a $G$-connection such that its restriction to each leaf $S_{c}$ is locally flat. Let $\Sigma$ be a smooth transverse section to the foliation induced by $D$ (for instance, we can parametrize $\Sigma$ by the transverse coordinate $c$ ). Consider the restriction $X_{\mid \Sigma}$ of the adapted frame $X=\left(X_{1}, \ldots, X_{n}\right)$ to $\Sigma$. Since the restriction of $\nabla$ to the leaves is flat, we can extend $X_{\mid \Sigma}$ to a local adapted frame $\bar{X}=\left(\bar{X}_{1}, \ldots, \bar{X}_{n}\right)$ defined on $U$ by parallel transport along the leaves, so that

$$
\nabla_{\bar{X}_{i}} \bar{X}_{j}=0, \text { if } 1 \leq i, j \leq k
$$

Using Frobenius's Theorem we get coordinates $x^{1}, \ldots, x^{n}$ such that $\bar{X}_{i}=\frac{\partial}{\partial x^{i}}$ for $i=1, \ldots, k$.

Since the uniqueness of $\tau_{c}$ implies that it varies smoothly with $c$, the following result is a consequence of the second point of Remark 3.7.

Corollary 3.11 If $\partial_{\mathfrak{h}}$ is injective then $P$ is integrable if and only if the distribution $D$ is involutive and the $H$-structures induced on leaves are all of them integrable.

We will treat the case in which $H$ is the following subgroup of $G l(2, \mathbb{R})$ :

$$
H=\left\{\left(\begin{array}{ll}
a & b \\
0 & c
\end{array}\right) \mid a^{\beta}=c^{\alpha}\right\},
$$

with $\alpha \beta \neq 0$ (see Example 2.13). This case will be very useful in the following section.

Proposition 3.12 With the above notations a G-structure is integrable if and only if the distribution $D$ is involutive.

Proof: We will show that every $H$-structure is integrable. Let $\mathfrak{h}$ be the Lie algebra of $H$ :

$$
\mathfrak{h}=\left\{\left(\begin{array}{cc}
\alpha a & b \\
0 & \beta a
\end{array}\right) \mid a, b \in \mathbb{R}\right\} .
$$

An easy calculation show that $\partial_{\mathfrak{h}}$ is surjective and

$$
\mathfrak{h}^{(1)}=\left\{\left(S_{1}, S_{2}\right) \mid S_{1}=b_{1} B, S_{2}=\frac{b_{1}}{\alpha} A+b_{2} B\right\}
$$

where

$$
A=\left(\begin{array}{ll}
\alpha & 0 \\
0 & \beta
\end{array}\right), \quad B=\left(\begin{array}{ll}
0 & 1 \\
0 & 0
\end{array}\right)
$$


is a basis of $\mathfrak{h}$. Let $X_{1}, X_{2}$ be an adapted local frame of the $H$-structure, and put $\left[X_{1}, X_{2}\right]=$ $\gamma_{1} X_{1}+\gamma_{2} X_{2}$. Since $\partial_{\mathfrak{h}}$ is surjective we find $\tau=\left(\tau_{i j}^{k}\right)$ such that $\partial_{\mathfrak{h}} \tau=\left(\gamma_{1}, \gamma_{2}\right)$. For instance, we can take

$$
\tau_{1}=\frac{\gamma_{2}}{\beta} A+\gamma_{1} B, \quad \tau_{2}=0 .
$$

Taking into account (15) and putting

$$
\left\{\begin{array}{l}
\Delta_{1}=\tau_{1}+S_{1}=\frac{\gamma_{2}}{\beta} A+\left(b_{1}+\gamma_{1}\right) B \\
\Delta_{2}=\tau_{2}+S_{2}=\frac{b_{1}}{\alpha} A+b_{2} B
\end{array}\right.
$$

the matrix differential equation

$$
X_{1}\left(\Delta_{2}\right)-X_{2}\left(\Delta_{1}\right)+\left[\Delta_{1}, \Delta_{2}\right]-\gamma_{1} \Delta_{1}-\gamma_{2} \Delta_{2}=0
$$

is equivalent to the system

$$
\left.\begin{array}{r}
X_{1}\left(\frac{b_{1}}{\alpha}\right)-X_{2}\left(\frac{\gamma_{2}}{\beta}\right)-\frac{\gamma_{1} \gamma_{2}}{\beta}-\frac{\gamma_{2} b_{1}}{\alpha}=0 \\
X_{1}\left(b_{2}\right)-X_{2}\left(b_{1}+\gamma_{1}\right)+(\alpha-\beta)\left(\frac{\gamma_{2} b_{2}}{\beta}-\frac{\left(b_{1}+\gamma_{1}\right) b_{1}}{\alpha}\right)-\gamma_{1}\left(b_{1}+\gamma_{1}\right)-\gamma_{2} b_{2}=0
\end{array}\right\}
$$

where $b_{1}$ and $b_{2}$ are the unknown functions. The above system of PDE's has always solution since the first equation does not involve the unknown function $b_{2}$. The solution of this system can be obtained by solving two ordinary differential equations. Therefore these solutions depend smoothly on some parameters. We conclude that all the $H$-structures are integrable, and from Proposition 3.10 any $G$-structure with a tangent $H$-structure is integrable if and only if the two-dimensional distribution is involutive.

Another interesting case is when $H$ is given by

$$
H=\left\{\left(\begin{array}{ll}
a & 0 \\
0 & c
\end{array}\right) \mid a^{\beta}=c^{\alpha}\right\},
$$

with $\alpha \beta \neq 0$. One show that in this case $\partial_{\mathfrak{h}}$ is bijective and from Corollary 3.11 we obtain the following.

Corollary 3.13 A G-structure of this type is integrable if and only if the distribution is involutive and the H-structures induced on the leaves are all of them integrable. The latter occurs if and only if the unique torsionless $H$-connection (that exists) on each leaf has zero curvature.

Another useful remark for our purposes is the following. 
Remark 3.14 In several cases $G$ is the intersection of $\bar{G}$ and $S l(n, \mathbb{R})$, thus, a $G$-structure $P$ will be obtained as intersection of a $\bar{G}$-structure $\bar{P}$ and a $S l(n, \mathbb{R})$-structure given by a volume form $\Omega$, with the compatibility condition that there exist on each point of $M$ an adapted frame $v_{1}, \ldots, v_{n}$ of $\bar{P}$ such that $\Omega\left(v_{1}, \ldots, v_{n}\right)=1$.

Concerning this situation we have the following result.

Proposition 3.15 A G-structure $P$ is integrable if and only if the $\bar{G}$-structure $\bar{P}$ is integrable and there exist local coordinates $x^{1}, \ldots, x^{n}$ adapted to $\bar{P}$ (i.e., $\left(\frac{\partial}{\partial x^{1}}, \ldots, \frac{\partial}{\partial x^{n}}\right) \in \bar{P}$ ) such that $\mathcal{L}_{\frac{\partial}{\partial x^{i}}} \Omega=0$ for all $i=1, \ldots, n$.

Unfortunately, this proposition is too difficult to apply in the form that it is stated because in order to show the integrability of $P$ we need to find a privileged local coordinate system adapted to $\bar{P}$. Therefore, we describe an alternative approach characterizing the integrability of $P$. Instead of expressing this condition in a privileged coordinate system adapted to $\bar{P}$ as in Proposition 3.15, we can reformulate it in terms of an arbitrary coordinate system $x^{1}, \ldots, x^{n}$ adapted to $\bar{P}$, i.e. such that $\left(\frac{\partial}{\partial x^{2}}\right) \in \bar{P}$. We write $\Omega=b\left(x^{1}, \ldots, x^{n}\right) d x^{1} \wedge \ldots \wedge d x^{n}$ and consider the $\bar{G}$-connection $\widetilde{\nabla}$ defined by $\widetilde{\nabla} \frac{\partial}{\partial x^{i}} \frac{\partial}{\partial x^{j}}=0$. Any torsion free $\bar{G}$-connection $\nabla$ can we written as $\nabla=\widetilde{\nabla}+\tau$, where $\tau: U \rightarrow \overline{\mathfrak{g}}^{(1)} \subset \operatorname{Hom}\left(\mathbb{R}^{\mathrm{n}}, \mathfrak{g}\right)$. ¿From Proposition 3.8. the vanishing of the curvature of $\nabla$ is equivalent to the system of PDE's:

$$
\frac{\partial \tau_{j}}{\partial x^{i}}-\frac{\partial \tau_{i}}{\partial x^{j}}+\left[\tau_{i}, \tau_{j}\right]=0 .
$$

Finally, $\nabla$ is a $G$-connection if and only if $\nabla \Omega=0$. Since

$$
\left(\nabla_{\frac{\partial}{\partial x^{i}}} \Omega\right)\left(\frac{\partial}{\partial x^{1}}, \ldots, \frac{\partial}{\partial x^{n}}\right)=\frac{\partial b}{\partial x^{i}}-\sum_{j=1}^{n} \Omega\left(\frac{\partial}{\partial x^{1}}, \ldots, \nabla_{\frac{\partial}{\partial x^{i}}} \frac{\partial}{\partial x^{j}}, \ldots, \frac{\partial}{\partial x^{n}}\right)=\frac{\partial b}{\partial x^{i}}-b \operatorname{tr}\left(\tau_{i}\right),
$$

we can characterize the equation $\nabla \Omega=0$ as $\frac{\partial \log (b)}{\partial x^{i}}=\operatorname{tr}\left(\tau_{i}\right)$ for all $i=1, \ldots, n$.

Remark 3.16 In several cases, straightforward computations show that if $\tau$ takes values in $\overline{\mathfrak{g}}^{(1)}$ then $\operatorname{tr}\left(\tau_{k}\right)=0$ for some $k$. Therefore, assuming that $\bar{P}$ is integrable, a necessary condition for the integrability of $P$ is that $b\left(x_{1}, \ldots, x_{n}\right)$ does not depend on $x^{k}$, or equivalently,

$$
\mathcal{L}_{\frac{\partial}{\partial x^{k}}} \Omega=0
$$




\section{Some examples of $G$-structures defined by tensors}

The main purpose of this paper is a systematic study of those $G$-structures associated to uniform elastic bodies. Before to do that, we will discuss $G$-structures defined by tensors of type $(r, s)$, with $r+s \leq 3$. The results will be useful in the next sections.

- If $F=T_{1}^{0} \mathbb{R}^{3}$ then, since $G l(3, \mathbb{R})$ acts transitively on $F$, we can take $u=e_{1}$. Therefore the isotropy group $G$ of $u$ consists of matrices of the form

$$
\left(\begin{array}{lll}
1 & a & b \\
0 & c & d \\
0 & e & f
\end{array}\right)
$$

The associated fiber bundle is $T M$. Thus, a $G$-structure is given by a vector field $X$ without zeros. Hence every $G$-structure is integrable, since we can always choose local coordinates $x^{1}, x^{2}, x^{3}$ such that $X=\frac{\partial}{\partial x^{1}}$.

- If $F=T_{0}^{1} \mathbb{R}^{3}$ then we can take $u=e^{1}$, and in this case $G$ is the group of matrices obtained by transposing (6). The associated fiber bundle is the cotangent bundle $T^{*} M$. Therefore a $G$-structure is given by a one-form $\omega$ without zeros. Its integrability is equivalent to the existence of local coordinates such that $\omega=d x^{1}$, i.e., $\omega$ is locally exact, or equivalently, $\omega$ is closed.

- If $F=T_{1}^{1} \mathbb{R}^{3}$, then the action of $G l(3, \mathbb{R})$ on $F$ is by conjugation, so that the corresponding orbits are not trivial: $u_{1}, u_{2} \in F$ are in the same orbit if and only if they have the same canonical form over $\mathbb{R}$. We will study the different possibilities in dimension 3. The minimum polynomial is one of the following types:

(a) $\left((x-\alpha)^{2}+\beta^{2}\right)(x-\lambda), \beta \neq 0$ with canonical form over $\mathbb{R}$

$$
\left(\begin{array}{ccc}
\alpha & \beta & 0 \\
-\beta & \alpha & 0 \\
0 & 0 & \lambda
\end{array}\right)
$$

(b) $(x-\lambda)(x-\mu)(x-\nu)$, where $\lambda, \mu, \nu$ are three different eigenvalues; in this case the Jordan form is diagonal.

(c) $(x-\lambda)^{2}(x-\mu), \lambda \neq \nu$ with Jordan form

$$
\left(\begin{array}{lll}
\lambda & 1 & 0 \\
0 & \lambda & 0 \\
0 & 0 & \mu
\end{array}\right)
$$


(d) $(x-\lambda)(x-\mu), \lambda \neq \nu$ with diagonal Jordan form

$$
\left(\begin{array}{ccc}
\lambda & 0 & 0 \\
0 & \lambda & 0 \\
0 & 0 & \mu
\end{array}\right)
$$

(e) $(x-\lambda)^{3}$, with Jordan form

$$
\left(\begin{array}{lll}
\lambda & 1 & 0 \\
0 & \lambda & 1 \\
0 & 0 & \lambda
\end{array}\right)
$$

(f) $(x-\lambda)^{2}$, with Jordan form

$$
\left(\begin{array}{ccc}
\lambda & 1 & 0 \\
0 & \lambda & 0 \\
0 & 0 & \lambda
\end{array}\right)
$$

(g) $(x-\lambda)$, in this case we have a homothetic transformation.

The isotropy groups of theses matrices consist of the matrices of the following form:
(a) $\left(\begin{array}{ccc}a & 0 & 0 \\ 0 & b & c \\ 0 & -c & b\end{array}\right)$
(b) $\left(\begin{array}{lll}a & 0 & 0 \\ 0 & b & 0 \\ 0 & 0 & c\end{array}\right)$
(c) $\left(\begin{array}{lll}a & b & 0 \\ 0 & a & 0 \\ 0 & 0 & c\end{array}\right)$
(d) $\left(\begin{array}{lll}a & 0 & 0 \\ 0 & b & c \\ 0 & d & e\end{array}\right)$
(e) $\left(\begin{array}{lll}a & b & c \\ 0 & a & b \\ 0 & 0 & a\end{array}\right)$
(f) $\left(\begin{array}{lll}a & 0 & b \\ c & d & e \\ 0 & 0 & d\end{array}\right)$

The case $(\mathrm{g})$ is trivial since the homothetic transformation commutes with every element in $G l(3, \mathbb{R})$.

Remark 4.1 We notice that the isotropy group is determined by the relations between $\lambda, \mu$ and $\nu$ and the fact that $\beta \neq 0$, but not by the particular values of them. For instance, we can take $\lambda=0, \mu=1, \nu=-1, \alpha=0$ and $\beta=1$. Then the non trivial orbits of $F=T_{1}^{1} M$ are given by $u \in F$ fulfilling one and only one of the following equations:

$$
u^{3}+u=0, u^{3}-u=0, u^{3}-u^{2}=0, u^{2}-u=0, u^{3}=0, u^{2}=0 .
$$


The associated fiber bundle is $T_{1}^{1} M=T M \otimes T^{*} M$. Therefore, a $G$-structure with $G$ a subgroup of the above list is given by a tensor field $h$ of type $(1,1)$ such that for each $x \in M h_{x}: T_{x} M \rightarrow T_{x} M$ has constant canonical form $u$. These $G$-structures were extensively treated in the literature. The tensor field $h$ is called a 0 -deformable vector one-form (or (1,1)-type tensor field) [17]. The general notion of 0-deformability can be found in [12]:

Definition 4.2 A section $\sigma$ of a vector bundle $\xi=(E, \pi, M, F)$ is said to be 0 deformable if for each $x, y \in M$ there exist a linear isomorphism $\alpha_{x, y}: F_{x} \rightarrow F_{y}$ such that $\alpha_{x, y}(\sigma(x))=\sigma(y)$.

Remark 4.3 (i) We notice the similarity of this definition with the notion of material uniformity of a body $B$ (see Section 5$)$.

(ii) A finite set of 0 -deformable cross-sections $\Sigma_{\xi}=\left(\sigma_{1}, \ldots, \sigma_{m}\right)$ of $\xi^{p_{i}, q_{i}}=\xi^{\otimes p_{i}} \otimes$ $\left(\xi^{*}\right)^{\otimes q_{i}}$ define a $\Sigma$-bundle (see [12]) where $\xi=(E, \pi, B, F)$ is a vector bundle. In fact, the definition of $\Sigma$-bundle involves:

(a) a smooth vector bundle $\xi=(E, \pi, B, F)$.

(b) a finite ordered set of cross-sections $\Sigma_{\xi}=\left(\sigma_{1}, \ldots, \sigma_{m}\right), \sigma_{i} \in \operatorname{Sec} \xi^{p_{i}, q_{i}}$,

subject to the following condition:

there are a finite ordered system $\Sigma_{F}=\left(u_{1}, \ldots, u_{m}\right)$ of tensors $u_{i} \in F^{p_{i}, q_{i}}$ and a coordinate representation $\left\{U_{\alpha}, \psi_{\alpha}\right\}$ of $\xi$ such that

$$
\psi_{\alpha}^{p_{i}, q_{i}}\left(x, u_{i}\right)=\sigma_{i}(x) \in \xi_{x}^{p_{i}, q_{i}}, \quad x \in U_{\alpha}, \quad i=1, \ldots, m .
$$

Then we will say that the section $\sigma_{i}$ is 0 -deformable to $u_{i}$. A $\Sigma$-bundle gives a reduction of the structure group from $G L(F)$ to an algebraic subgroup. If $\xi$ is the tangent bundle we obtain in this way a $G$-structure with $G$ an algebraic subgroup of $G l(n, \mathbb{R})$.

Coming back to the case of a 0-deformable vector one-form $h$ we are interested in the characterization of the integrability of the $G$-structure defined by $h$. The following theorem was proved by E.T. Kobayashi [14] (see also [17]).

Theorem 4.4 Let $h$ be a 0-deformable vector one-form on a manifold $M$, with characteristic polynomial

$$
\prod_{i} p_{i}(x)^{d_{i}}
$$


where $p_{i}(x)$ are irreducible and coprime polynomials in $x$ over $\mathbb{R}$. The minimum polynomial is

$$
\prod_{i} p_{i}(x)^{v_{i}}
$$

We assume that for each $i$ we have $v_{i}=1$ or $v_{i}=d_{i}$. Then the $G$-structure defined by $h$ is integrable if the Nijenhuis tensor of $h, H_{h}$ is zero, where $N_{h}$ is a vector 2-form defined by

$$
\frac{1}{2} N_{h}(X, Y)=[h X, h Y]-h[h X, Y]-h[X, h Y]+h^{2}[X, Y] .
$$

Remark 4.5 (i) In dimension three the above theorem gives a suficient condition for the integrability in all the cases except when $u^{2}=0$.

(ii) $N_{h}=0$ is always a necessary condition for the integrability.

- If $F=T_{0}^{2} \mathbb{R}^{3}=S^{2} \mathbb{R}^{3} \oplus \bigwedge^{2} \mathbb{R}^{3}$, the action of an element $A \in G l(3, \mathbb{R})$ on $f \in F$ is given by $A^{t} f A$. There are two fundamental cases that we will discuss separately:

- $u \in S^{2} \mathbb{R}^{3}$, then applying Sylvester's Theorem we deduce that $u$ is in the orbit of $\epsilon_{1} e^{1} \otimes e^{1}+\epsilon_{2} e^{2} \otimes e^{2}+\epsilon_{3} e^{3} \otimes e^{3}$ for some $\epsilon_{1}, \epsilon_{2}, \epsilon_{3} \in\{-1,0,1\}$, i.e., the range and the signature determine the orbit of $u$. The associated fiber bundle is $T_{0}^{2} M$, and a $G$-structure with $G$ the isotropy group of $u$ is given by a symmetric $(0,2)$-tensor field which is 0-deformable to $u$. If $u=e^{1} \otimes e^{1}+e^{2} \otimes e^{2} \pm e^{3} \otimes e^{3}$ we obtain, respectively, a Riemannian or Lorentzian metric $g$ on $M$. The integrability of this structure is characterized by the vanishing of the scalar curvature of $g$, but, in dimension three, this is equivalent to the vanishing of the Ricci tensor of $g$.

- $u \in \bigwedge^{2} \mathbb{R}^{3} \backslash\{0\}$. By Darboux's Theorem $u$ is in the orbit of $e^{1} \wedge e^{2}$. Therefore a $G$-structure with

$$
G=\left\{\left(\begin{array}{cc}
A & 0 \\
v & c
\end{array}\right) \mid A \in S l(2, \mathbb{R}), c \in \mathbb{R}^{*}\right\}
$$

is given by a two-form $\eta$ without zeros. The integrability is also characterized by Darboux's Theorem: The $G$-structure is integrable if and only if $d \eta=0$.

- The case $F=T_{2}^{0} \mathbb{R}^{3}$ is formally analogous to the above case, but the integrability condition is different.

- Finally, we will consider the case $F=\wedge^{3} \mathbb{R}^{3} \subset T_{0}^{3} \mathbb{R}^{3}$. We can take $u=e^{1} \wedge e^{2} \wedge e^{3}$, $G=G_{u}=S l(3, \mathbb{R})$ and $Q=\Omega^{3}(M)$. Therefore a $G$-structure over $M$ is given by a three-form without zeros, i.e., a volume form $\Omega$ on $M$. As we know, every $S l(3, \mathbb{R})$ structure is integrable. 


\section{Uniformity and homogeneity of simple materials}

A body $B$ is a 3-dimensional differentiable manifold which can be covered with just one chart. An embedding $\Phi: B \longrightarrow \mathbb{R}^{3}$ is called a configuration of $B$. The body is identified with any one of its configurations, say $\Phi_{0}: B \longrightarrow \mathbb{R}^{3}$, called a reference configuration. Given any

arbitrary configuration $\Phi: B \longrightarrow \mathbb{R}^{3}$, the change of configurations $\kappa=\Phi \circ \Phi_{0}^{-1}$ is called a deformation. We fix a reference configuration $\Phi_{0}$ and, from now on, $B$ and its image $\Phi_{0}(B)$ will be identified. The mechanical behaviour of a hyperelastic material body is characterized by one function $W$ which depends, at each point of $B$, only on the value of the derivative of the deformation evaluated at that point (see [25, 26, 27, 31, 32, 33, 34]). $W$ measures the strain energy per unit volume of reference configuration. In a more general material bodies, $W$ can depend also of higher order gradients or even more complicated microstructures (see [18, 19, 20, 21, 22].

$B$ is said to be materially uniform if for two arbitrary points $X, Y \in B$ there exists a local diffeomorphism $\phi$ from a neighbourhood of $X$ onto a neighbourhood of $Y$ such that $\phi(X)=Y$ and

$$
W\left(j_{Y, \kappa(Y)}^{1} \kappa\right)=W\left(j_{Y, \kappa(Y)}^{1} \kappa \cdot j_{X, Y}^{1} \phi\right)
$$

for all $j_{Y, \kappa(Y)}^{1} \kappa$. The 1-jet $j_{X, Y}^{1} \phi$ will be called a material 1-jet. It should be noticed that $j_{X, Y}^{1} \phi$ may be identified with the linear isomorphism $d \phi(X): T_{X} B \longrightarrow T_{Y} B . d \phi(X)$ is usually called a material isomorphism ([32]).

Denote by $\Omega(B)$ the collection of all material 1-jets. Thus, $\Omega(B) \subset \Pi^{1}(B, B)$, where $\Pi^{1}(B, B)$ is the Lie groupoid of all invertible 1-jets on the manifold $B$ (see [24] for a general reference on Lie groupoids). We have canonical mappings $\alpha: \Pi^{1}(B, B) \longrightarrow B$ and $\beta: \Pi^{1}(B, B) \longrightarrow B$ defined by

$$
\alpha\left(j_{X, Y}^{1} \phi\right)=X, \beta\left(j_{X, Y}^{1} \phi\right)=Y,
$$

respectively. Their restrictions to $\Omega(B)$ will be denoted by the same symbols. A direct computation from (77) shows the following result.

Proposition 5.1 If $B$ is uniform, then $\Omega(B)$ is a groupoid with source and target mappings $\alpha$ and $\beta$, respectively. In fact, $\Omega(B)$ is a subgroupoid of the Lie groupoid $\Pi^{1}(B, B)$ of all invertible 1-jets on the manifold $B$.

Definition $5.2 A$ material symmetry at a point $X$ is a 1-jet $j_{X, X}^{1} \phi$ of a local diffeomorphism at $X$ such that

$$
W\left(j_{X, \kappa(X)}^{1} \kappa\right)=W\left(j_{X, \kappa(X)}^{1} \kappa \cdot j_{X, X}^{1} \phi\right)
$$

for all $j_{X, \kappa(X)}^{1} \kappa$. 
¿From (8) we deduce that the collection $G(X)$ of all material symmetries at $X$ has a structure of group which is called the symmetry group at $X$. If $j_{X, Y}^{1} \phi$ is a material 1-jet joining two points $X$ and $Y$, we deduce that the symmetry groups at $X$ and $Y$ are conjugate:

$$
G(Y)=j_{X, Y}^{1} \phi \circ G(X) \circ\left(j_{X, Y}^{1} \phi\right)^{-1}
$$

Definition 5.3 We say that $B$ enjoys smooth uniformity if $\Omega(B)$ is a Lie groupoid, which will be called the material Lie groupoid.

In such a case, there exist local sections of the projection $(\alpha, \beta): \Omega(B) \longrightarrow B \times B$ given by $(\alpha, \beta)\left(j_{X, Y}^{1} \phi\right)=(X, Y)$. Such a local section, say $P: B \times B \longrightarrow \Omega(B)$ assigns to each pair $(X, Y)$ of material points a material 1-jet connecting them. Such a section $P$ is called a local material uniformity. If there exists a global section of $(\alpha, \beta)$, then $B$ enjoys smooth global uniformity, or, equivalently, the Lie groupoid $\Omega(B)$ is smoothly transitive.

By applying well-known results on Lie groupoids and frame bundles, we get the following (see [11]).

Proposition 5.4 If $B$ enjoys smooth uniformity, then:

(i) $G\left(X_{0}\right)$ is a Lie group.

(ii) $\alpha^{-1}\left(X_{0}\right)$ is a principal $G\left(X_{0}\right)$-bundle over $B$ whose canonical projection is the restriction of $\beta$.

Proof: (i) Since $(\alpha, \beta)$ is a submersion, we deduce that $G\left(X_{0}\right)=(\alpha, \beta)^{-1}\left(X_{0}\right)$ is a closed submanifold of $\Omega(B)$. Hence, it is a Lie group.

(ii) First of all, since $\alpha$ is a submersion, we deduce that $\alpha^{-1}\left(X_{0}\right)$ is a closed submanifold of $\Omega(B)$. Moreover, since $(\alpha, \beta)$ is a submersion, there exist an open covering $\left\{U_{a}\right\}$ of $B$ and local sections $\sigma_{a, b}: U_{a} \times U_{b} \longrightarrow \Omega(B)$ of $(\alpha, \beta)$. If $X_{0} \in U_{a_{0}}$, we define

$$
\sigma_{a}(X)=\sigma_{a_{0}, a}\left(X_{0}, X\right), \text { for } X \in U_{a}
$$

In other words, $\sigma_{a}$ assigns (in a differentiable way) to each material point $X$ a material 1-jet connecting $X_{0}$ and $X$. Thus, we have obtained a family of local sections $\left\{\sigma_{a}\right\}$ of $\beta: \alpha^{-1}\left(X_{0}\right) \longrightarrow B$ which define a principal $G\left(X_{0}\right)$-bundle.

Next, we fix a point $X_{0}$ at $B$. The tangent bundle $T_{X_{0}} B$ is a linear approximation of an infinitesimal piece of material around $X_{0}$. But $T_{X_{0}} B$ is completely characterized by a basis. This fact leads us to the following definition. 
Definition 5.5 A linear frame $Z_{0}$ at a material point $X_{0}$ will be called a reference crystal (at $\left.X_{0}\right)$.

A reference crystal $Z_{0}$ is just a 1 -jet $j_{0, X_{0}}^{1} \psi$ of a local diffeomorphism from $0 \in \mathbb{R}^{3}$ into $X_{0}$. Thus, we can transport $Z_{0}$ to any point of $B$ by composing it with smooth material uniformities. The next result is also standard in the literature (see [11]).

Theorem 5.6 (i) $G=Z_{0}^{-1} \circ G\left(X_{0}\right) \circ Z_{0}$ is a Lie subgroup of $G l(3, \mathbb{R})$.

(ii) Denote by $\omega(B)$ the set of all linear frames at all the point of $B$ obtained by translating $Z_{0}$. Then $\omega(B)$ is a $G$-structure on $B$.

Proof: (i) Put $\left.G L\left(B, X_{0}\right)=\left\{j_{X_{0}, X_{0}}^{1} \phi\right\} \subset \Pi^{1}(B, B)\right\} . G L\left(B, X_{0}\right)$ is a Lie group which is isomorphic to $G l(3, \mathbb{R})$. Since the mapping

$$
G L\left(B, X_{0}\right) \longrightarrow G l(3, \mathbb{R}), j_{X_{0}, X_{0}}^{1} \phi \leadsto Z_{0}^{-1} \circ j_{X_{0}, X_{0}}^{1} \phi \circ Z_{0},
$$

is smooth, it follows that $G=Z_{0}^{-1} \circ G\left(X_{0}\right) \circ Z_{0}$ is a Lie subgroup of $G l(3, \mathbb{R})$.

(ii) Take the family of local sections $\left\{\sigma_{a}\right\}$ obtained in Proposition 5.4 A family of local sections $\left\{\tau_{a}\right\}$ of $\omega(B)$ for the open covering $\left\{U_{a}\right\}$ is obtained as follows:

$$
\tau_{a}(X)=\sigma_{a}(X) \circ Z_{0}, \text { for } X \in U_{a} .
$$

An straightforward computation shows that $\omega(B)$ is in fact a $G$-reduction of $\mathcal{F B}$.

This $G$-structure will be called material.

Remark 5.7 (1) If we perform a change of reference configuration, the $G$-structure remains the same, provided that the point $X_{0}$ and the reference crystal $Z_{0}$ are dragged by the change of configuration.

(2) If we choose a different point $X_{0}^{\prime}$, we obtain the same $G$-structure provided that the reference crystal is the one obtained using a material uniformity from $X_{0}$ to $X_{0}^{\prime}$.

(3) If we change the reference crystal $Z_{0}$ to $Z_{0}^{\prime}=Z_{0} A$, where $A \in G l(3, \mathbb{R})$, we obtain a conjugate $G$-structure $\omega(B) A$, with conjugate structure group $A^{-1} G A$.

Definition 5.8 $A$ body $B$ is said to be homogeneous if there exists a global deformation $\kappa$ such that $Q$ defined by

$$
Q(X)=j_{0, X}^{1}\left(\kappa^{-1} \circ \tau_{\kappa(X)}\right), \forall X \in B,
$$

is a uniform reference, where $\tau_{\kappa(X)}: \mathbb{R}^{3} \rightarrow \mathbb{R}^{3}$ denotes the translation by $\kappa(X)$.

$B$ is said to be locally homogeneous if around each point $X$ of $B$ there exists an open neighbourhood $U$ which is homogeneous. 
The following result gives a geometric characterization of the local homogeneity.

Theorem 5.9 B is locally homogeneous if and only if the associated material G-structure is integrable.

Remark 5.10 According to Remark 5.7 the above definition does not depend on the chosen crystal reference.

\section{Classification of material $G$-structures}

Our purpose is to study systematically the possible material $G$-structures associated to elastic bodies.

For physical reasons (see 32, 34]) we are only interested in $G$-structures with $G$ a Lie subgroup of the special linear group $S l(3, \mathbb{R})$. The first step is to classify the subgroups of $S l(3, \mathbb{R})$. A classification modulo conjugation is usually attributed to S. Lie [23, 28, 32, 34]. We reproduce here the list as it is presented in Wang [34. This list gives the classification of the Lie subalgebras of the Lie algebra $\operatorname{sl}(3)$ of $S l(3, \mathbb{R})$ and their corresponding connected Lie subgroups, see Appendix A.

There are three types of solids, namely:

- isotropic solids belong to type 16,

- transversely isotropic solids belong to type 8 with parameter $\alpha=0$, and

- crystalline solids belong to type 5 with $\alpha=\beta=\gamma=0$.

All other types are fluid crystals. For instance,

- isotropic fluids belong to type 9, and

- fluid crystal of first kind (respectively, second kind) belong to type 11 (respectively, $10)$.

The first five families consist of three fundamental types, denoted by A, B and C, respectively:

- type $\mathrm{A}$ is characterized by considering $\alpha, \beta$ and $\gamma$ as variables and they are algebraic subgroups. 
- type $\mathrm{B}$ is characterized by considering $\alpha, \beta$ and $\gamma$ as fixed parameters, with $(\alpha, \beta, \gamma) \neq$ $(0,0,0)$ and $\alpha+\beta+\gamma=0$. They define an element $[\alpha, \beta]$ of $\mathbb{P R}^{1}$. The corresponding subgroup of the list is not algebraic, but it is contained in a bigger "natural" algebraic subgroup if the parameters are integer.

- type $\mathrm{C}$ is obtained by taking $\alpha=\beta=\gamma=0$ and all of them are algebraic.

The types 6-8 contain two different cases:

- type $\mathrm{A}$ is characterized by taking $\alpha$ and $\beta$ as variables. They are algebraic subgroups.

- type $\mathrm{B}$ is characterized by taking $\alpha$ and $\beta$ as fixed parameters. They are algebraic if and only if $\alpha=0$.

The other families consist of a unique type, and they are algebraic except families 17, 18, 21, 22 and 25.

In what follows, we will discuss the $G$-structures with $G$ an algebraic subgroup of $S l(3, \mathbb{R})$. We remark that the Lie subgroups included in the list are connected, however we will consider the corresponding natural algebraic subgroups.

Given a such $G$-structure, and according to Remark 3.14 sometimes we will consider an enlarged structure $\bar{P}$ with structural group $\bar{G} \subset G l(3, \mathbb{R})$ such that $\bar{G} \cap S l(3, \mathbb{R})=G$. The relation between the integrability of $P$ and $\bar{P}$ is given by Proposition 3.15 . Thus, in some cases we have only characterized the integrability of $\bar{P}$.

\section{The group 1A}

The group $G_{1 A}$ is just the isotropy group of the linear subspaces $\left\langle e_{3}\right\rangle \subset\left\langle e_{2}, e_{3}\right\rangle$ and the tensor $w=e^{1} \wedge e^{2} \wedge e^{3}$ on $\mathbb{R}^{3}$. Then such a $G_{1 A^{-}}$-structure $P$ is given by a one-dimensional distribution $L$, a two-dimensional distribution $D$ with $L \subset D$, and a volume form $\Omega$.

Proposition 6.1 $P$ is integrable if and only if $D$ is involutive.

Proof: If $D$ is involutive and $X, Y, Z$ is an adapted local basis, i.e., $L=\langle X\rangle, D=\langle X, Y\rangle$, then we have $[X, Y]=\alpha X+\beta Y$. Therefore there exist local functions $f$ and $g$ such that $[f X, g Y]=0$. Thus, there are local coordinates $y^{1}, y^{2}, y^{3}$ such that $f X=\frac{\partial}{\partial y^{1}}$ and $g Y=\frac{\partial}{\partial y^{2}}$. If $\Omega=b\left(y^{1}, y^{2}, y^{3}\right) d y^{1} \wedge d y^{2} \wedge d y^{3}$, we define new coordinates $x^{1}=\int b\left(y^{1}, y^{2}, y^{3}\right) d y^{1}, x^{2}=y^{2}$, $x^{3}=y^{3}$, and hence $\left(x^{1}, x^{2}, x^{3}\right)$ are adapted coordinates. The converse is trivial.

\section{The group 1B}


As we have said before we only discuss the algebraic case. Thus, $\alpha, \beta$ and $\gamma$ are integer parameters. We can only consider the following six cases:

- $\alpha, \gamma>0$, then the tensor

$$
t=\underbrace{e^{1} \otimes \cdots \otimes e^{1}}_{\gamma} \otimes \underbrace{e_{3} \otimes \cdots \otimes e_{3}}_{\alpha}
$$

is invariant with respect to the natural action of $G L(3, \mathbb{R})$. In fact, the isotropy group of $t$ and $w$ is just the group $G_{1 B}$. Therefore, in this case a $G_{1 B}$-structure $P$ is given by a tensor field $T$ of type $(\gamma, \alpha)$ which is 0 -deformable to $t$, and a volume form $\Omega$. In addition we have a two-dimensional distribution $D$ on $B$ such that $T$ is tangent to $D$. ¿From Propositions 3.12 and 3.15 , we deduce the following.

Proposition 6.2 The $\bar{G}_{1 B}$-structure $\bar{P}$ is integrable if and only if the distribution $D$ is involutive. In this case, let us denote by $\left(x^{1}, x^{2}, x^{3}\right)$ local coordinates adapted to $\bar{P}$. If $P$ is integrable then necessarily $\mathcal{L}_{\frac{\partial}{\partial x^{2}}} \Omega=\mathcal{L}_{\frac{\partial}{\partial x^{3}}} \Omega=0$.

Proof: It only remains to prove the last assertion, which follows directly from Remark 3.16 and the following computation of $\overline{\mathfrak{g}}^{(1)}=\operatorname{ker} \partial_{\overline{\mathfrak{g}}}$, where $\partial_{\overline{\mathfrak{g}}}: \operatorname{Hom}\left(\mathbb{R}^{3}, \overline{\mathfrak{g}}\right) \rightarrow$ $\operatorname{Hom}\left(\bigwedge^{2} \mathbb{R}^{3}, \mathbb{R}^{3}\right)$ is given by $\partial_{\overline{\mathfrak{g}}}\left(\left(\tau_{i}\right)\right)=\left(\tau_{i j}^{k}-\tau_{j i}^{k}\right)$ and $\tau_{i}=\left(\begin{array}{ccc}\alpha a_{i} & 0 & 0 \\ b_{i} & \beta a_{i} & 0 \\ c_{i} & d_{i} & \gamma a_{i}\end{array}\right) \cdot$ ¿From the following table,

\begin{tabular}{c|c|c|c|} 
& $\tau_{12}^{k}-\tau_{21}^{k}$ & $\tau_{13}^{k}-\tau_{31}^{k}$ & $\tau_{23}^{k}-\tau_{32}^{k}$ \\
\hline$k=1$ & $0-\alpha a_{2}$ & $0-\alpha a_{3}$ & $0-0$ \\
\hline$k=2$ & $\beta a_{1}-b_{2}$ & $0-b_{3}$ & $0-\beta a_{3}$ \\
\hline$k=3$ & $d_{1}-c_{2}$ & $\gamma a_{1}-c_{3}$ & $\gamma a_{2}-d_{3}$ \\
\hline
\end{tabular}

we deduce that if $\tau=\left(\tau_{i}\right)_{i=1}^{3} \in \overline{\mathfrak{g}}^{(1)}$, then $a_{2}=a_{3}=0$ and therefore $\operatorname{tr} \tau_{2}=\operatorname{tr} \tau_{3}=0$.

- $\beta, \gamma>0$, then

$$
t=\underbrace{e^{2} \otimes \cdots \otimes e^{2}}_{\gamma} \otimes \underbrace{e_{3} \otimes \cdots \otimes e_{3}}_{\beta}
$$

is a tensor of type $(\gamma, \beta)$ defined on the subspace $\left\langle e_{2}, e_{3}\right\rangle$. Our group is just the isotropy group of $t$ and $w$. A $G$-structure is now given by a two-dimensional distribution $D$ and a tangent tensor field $D$ of type $(\gamma, \beta)$ which is 0 -deformable to $t$. The integrability condition is the same as in the precedent case. However, it should be noticed that now $T$ is not a global tensor field on $B$. 
- $\alpha, \beta>0$, then the subspace $\left\langle e_{3}\right\rangle$ and the transverse tensor

$$
t=\underbrace{u^{1} \otimes \cdots \otimes u^{1}}_{\beta} \otimes \underbrace{u_{2} \otimes \cdots \otimes u_{2}}_{\alpha}
$$

where $u_{1}, u_{2}$ is a basis of the quotient vector space $\mathbb{R}^{3} /\left\langle e_{3}\right\rangle$, together with the three-form $w$ determine the group $G_{1 B}$. Therefore a $G_{1 B}$-structure $P$ is, in this case, given by a one-dimensional distribution $L$, a transverse tensor field $T$ to $L$ which is 0 -deformable to $t$, and a volume form $\Omega$.

Proposition 6.3 $\bar{P}$ is integrable if and only if it is projectable, i.e., the coefficients of $T$ in a system of coordinates $x^{1}, x^{2}, x^{3}$ adapted to the foliation induced by $L$ does not depend on the tangent coordinate $x^{3}$. Moreover, assume that $\left(x_{1}, x_{2}, x_{3}\right)$ are local coordinates adapted to $\bar{P}$ and $P$ is integrable. Then $\mathcal{L}_{\frac{\partial}{\partial x^{2}}} \Omega=\mathcal{L}_{\frac{\partial}{\partial x^{3}}} \Omega=0$.

Remark 6.4 An alternative description of $P$ is a tangent $H$-structure on a twodimensional distribution as in the precedent case, so that we can also apply Proposition 6.2

- $\alpha=0, \beta, \gamma \neq 0$, then we have that $e^{1},\left\langle e_{3}\right\rangle$ and $w$ determine $G_{1 B}$ as the isotropy group. Therefore, a $G_{1 B}$-structure $P$ is given by an one-form $\omega$ without zeros, a onedimensional distribution $L$ such that $\left.\omega\right|_{L}=0$, and a volume form $\Omega$.

Proposition 6.5 $P$ is integrable if and only if $\omega$ is closed.

Proof: If $d \omega=0$ then we have that the two-dimensional distribution $D=\{\omega=0\}$ is involutive, and, by a similar argument as in Proposition 6.1, there are local coordinates $\left(y^{1}, y^{2}, y^{3}\right)$ such that $L=\left\langle\frac{\partial}{\partial y^{3}}\right\rangle, D=\left\langle\frac{\partial}{\partial y^{2}}, \frac{\partial}{\partial y^{1}}\right\rangle$ and $\Omega=d y^{1} \wedge d y^{2} \wedge d y^{3}$. Thus $\omega=\lambda d y^{1}$. Since $\omega$ is closed, we deduce that $\lambda=\lambda\left(y^{1}\right)$ and, by defining $x^{1}=\int \lambda\left(y^{1}\right) d y^{1}$, $x^{2}=\frac{y^{2}}{\lambda\left(y^{1}\right)}$ and $x^{3}=y^{3}$, we obtain adapted coordinates $\left(x^{1}, x^{2}, x^{3}\right)$. The converse is trivial.

- $\beta=0, \alpha, \gamma \neq 0$, then $G_{1 B}$ is the isotropy group of the vector subspace $\left\langle e_{1}, e_{3}\right\rangle$, the tangent covector $e_{2}$ in this subspace, and the tensor $w$. Thus, a $G_{1 B}$-structure $P$ is given by a two-dimensional distribution $D$, a tangent one-form $\left.\omega\right|_{D}$ on $D$, and a volume form $\Omega$.

Proposition 6.6 $P$ is integrable if and only if $D$ is involutive and $\left.d \omega\right|_{D}=0$. 
Proof: As in Proposition 6.6.

- $\gamma=0, \alpha, \beta \neq 0$, then the group $G_{1 B}$ is the isotropy group of $e_{3}$, the subspace $\left\langle e_{2}, e_{3}\right\rangle$ and the tensor $w$. Therefore, in this case, a $G_{1 B}$-structure $P$ is given by a two-dimensional distribution $D$, a vector field $X$ without zeros belonging to $D$, and a volume form $\Omega$.

Proposition 6.7 $P$ is integrable if and only if $D$ is involutive and $\mathcal{L}_{X} \Omega=0$.

Proof: As in Proposition 6.1.

\section{The group $1 \mathrm{C}$}

The group $G_{1 C}$ is the isotropy group of the tensors $e_{3}, e^{1}$ and $w$ on $\mathbb{R}^{3}$. Therefore a $G_{1 C^{-}}$ structure $P$ is given by a one-form $\omega$, a vector field $X$ such that $\omega(X)=0$, and a volume form $\Omega$.

Proposition 6.8 $P$ is integrable if and only if $\omega$ is closed and $\mathcal{L}_{X} \Omega=0$.

Proof: If $d \omega=0$ then the two-dimensional distribution $D=\{\omega=0\}$ is involutive. Take a vector field $Y$ such that $D=\langle X, Y\rangle$. Since $D$ is involutive, there are functions $f$ and $g$, $g \neq 0$, such that $Y^{\prime}=f X+g Y$ verifies $\left[X, Y^{\prime}\right]=0$. Thus, there exist local coordinates $\left(y^{1}, y^{2}, y^{3}\right)$ such that $X=\frac{\partial}{\partial y^{2}}$ and $Y^{\prime}=\frac{\partial}{\partial y^{3}}$. We deduce that $\omega=\lambda d y^{1}$. Since $\omega$ is closed, $\lambda=\lambda\left(y^{1}\right)$ and we can assume without loss of generality that $\lambda=1$ (otherwise we only need to make a new change of coordinates). Now $\Omega=b\left(y^{1}, y^{2}, y^{3}\right) d y^{1} \wedge d y^{2} \wedge d y^{3}$, and by applying the argument in Proposition 6.1 we conclude. The converse is trivial.

\section{The group $2 \mathrm{~A}$}

The group $G_{2 A}$ is the isotropy group of the subspaces $\left\langle e_{3}\right\rangle,\left\langle e_{2}\right\rangle$ and of the tensor $w$. Thus, a $G_{2 A}$-structure $P$ is given by two transverse one-dimensional distributions $L_{1}$ and $L_{2}$ and a volume form $\Omega$.

Assume that the two-dimensional distribution $L_{1} \oplus L_{2}$ is involutive. Given an adapted local basis $Y_{1}, Y_{2}, Y_{3}$, i.e., such that $Y_{i} \in L_{i}$ for $i=1,2$ and $\Omega\left(Y_{1}, Y_{2}, Y_{3}\right)=1$, we define

$$
\tau\left(Y_{1}, Y_{2}, Y_{3}\right)=Y_{1}(\alpha)+Y_{2}(h)+\alpha h-\alpha \beta,
$$

where $\left[Y_{1}, Y_{2}\right]=\alpha Y_{1}+\beta Y_{2}$ and $\mathcal{L}_{Y_{1}} \Omega=h \Omega$. Given another adapted local basis $Y_{1}^{\prime}=f_{1} Y_{1}$, $Y_{2}^{\prime}=f_{2} Y_{2}$, and $Y_{3}^{\prime}=\frac{1}{f_{1} f_{2}} Y_{3}$, a direct computation shows that

$$
\tau\left(Y_{1}^{\prime}, Y_{2}^{\prime}, Y_{3}^{\prime}\right)=f_{1} f_{2} \tau\left(Y_{1}, Y_{2}, Y_{3}\right)
$$


Proposition 6.9 $P$ is integrable if and only if the two-dimensional distribution $L_{1} \oplus L_{2}$ is involutive and $\tau\left(Y_{1}, Y_{2}, Y_{3}\right)$ vanishes for an arbitrary adapted local frame $Y_{1}, Y_{2}, Y_{3}$.

Proof: One direction is obvious. For the other direction, we only need to prove that given an adapted local frame $Y_{1}, Y_{2}, Y_{3}$ as above there exist local functions $\lambda_{1}$ and $\lambda_{2}$ such that $\left[\lambda_{1} Y_{1}, \lambda_{2} Y_{2}\right]=0$ and $\mathcal{L}_{\lambda_{1} Y_{1}} \Omega=0$. To prove this we proceed as follows. If $\left[Y_{1}, Y_{2}\right]=\alpha Y_{1}+\beta Y_{2}$ and $\mathcal{L}_{Y_{1}} \Omega=h \Omega$, then

$$
Y_{1}\left(\lambda_{2}\right)+\beta \lambda_{2}=0, \quad Y_{2}\left(\lambda_{1}\right)-\alpha \lambda_{1}=0, \quad Y_{1}\left(\lambda_{1}\right)+h \lambda_{1}=0 .
$$

The first equation can always be integrated and the compatibility condition for the last two equations is just

$$
\begin{aligned}
(-\alpha h+\beta \alpha) \lambda_{1} & =\alpha Y_{1}\left(\lambda_{1}\right)+\beta Y_{2}\left(\lambda_{1}\right) \\
& =\left[Y_{1}, Y_{2}\right] \lambda_{1} \\
& =Y_{1} Y_{2} \lambda_{1}-Y_{2} Y_{1} \lambda_{1} \\
& =Y_{1}\left(\alpha \lambda_{1}\right)+Y_{2}\left(h \lambda_{1}\right) \\
& =\left(Y_{1} \alpha+Y_{2} h\right) \lambda_{1}+\alpha Y_{1} \lambda_{1}+h Y_{2} \lambda_{1} \\
& =\left(Y_{1} \alpha+Y_{2} h\right) \lambda_{1} .
\end{aligned}
$$

To end the proof, we remark that if $\left[\lambda_{1} Y_{1}, \lambda_{2} Y_{2}\right]=0$ and $\mathcal{L}_{\lambda_{1} Y_{1}} \Omega=0$, then there exist local coordinates $\left(y^{1}, y^{2}, y^{3}\right)$ such that $\lambda_{1} Y_{1}=\frac{\partial}{\partial y^{1}}, \lambda_{2} Y_{2}=\frac{\partial}{\partial y^{2}}, \mathcal{L}_{\frac{\partial}{\partial y^{1}}} \Omega=0, \mathcal{L}_{\frac{\partial}{\partial y^{2}}} \Omega=0$, and now, after an appropriate change of coordinates, we conclude.

\section{The group 2B}

We can only consider the algebraic case, i.e., with $\alpha, \beta$ and $\gamma$ integer parameters.

- If $\alpha \beta \gamma \neq 0$ then we have a tangent $H$-structure defined by tangent tensors to the vector subspace $\left\langle e_{1}, e_{2}\right\rangle$. We can consider the following two subcases:

- If $\beta$ and $\gamma$ are both positive, we have

$$
\underbrace{e^{1} \otimes \cdots \otimes e^{1}}_{\gamma} \otimes \underbrace{e_{2} \otimes \cdots \otimes e_{2}}_{\beta} \text { and } \underbrace{e^{2} \otimes \cdots \otimes e^{2}}_{\beta} \otimes \underbrace{e_{1} \otimes \cdots \otimes e_{1}}_{\gamma}
$$

as invariant tangent tensors of $G_{2 B}$.

- If $\beta>0$ and $\gamma<0$, then

$$
(\underbrace{e_{1} \otimes \cdots \otimes e_{1}}_{-\gamma}) \odot(\underbrace{e_{2} \otimes \cdots \otimes e_{2}}_{\beta})
$$

is an invariant tangent tensor. 
Thus, the group $G_{2 B}$ is the intersection of $G_{2 A}$ with the isotropy group of the above tangent tensors. Therefore, a $G_{2 B}$-structure $P$ is given by a one-dimensional distribution $L$, a two-dimensional distribution $D$ with $L \subset D$, a tangent tensor field on $D$ which is 0-deformable to the above tensors and a volume form.

¿From Proposition 3.10 and Corollaries 3.11] and 3.13. we have the following.

Proposition 6.10 $\bar{P}$ is integrable if and only if $D$ is involutive and the $H$-structures induced on the leaves of the foliation defined by $D$ are all of them integrable (the last condition occurs if and only if the unique torsion free $H$-connection on each leaf has zero curvature). Assume that $\bar{P}$ is integrable with adapted local coordinates $\left(x^{1}, x^{2}, x^{3}\right)$. If $P$ is integrable then $\mathcal{L}_{\frac{\partial}{\partial x^{2}}} \Omega=\mathcal{L}_{\frac{\partial}{\partial x^{3}}} \Omega=0$.

Proof: It only remains to prove the last assertion, which follows from Remark 3.16 and the computations made in the proof of Proposition 6.2 .

- If $\alpha \beta \gamma=0$ we consider the following subcases:

- $\alpha=0$, a $G_{2 B}$-structure $P$ is given by two one-dimensional distributions $L_{1}, L_{2}$, a 1-form $\omega$ such that $L_{1} \oplus L_{2}=\{\omega=0\}$ and a volume form $\Omega$.

Proposition 6.11 $P$ is integrable if and only if the conditions in Proposition 6.9 hold and $d \omega=0$.

Proof: ¿From Proposition 6.9 we conclude that there exist local coordinates

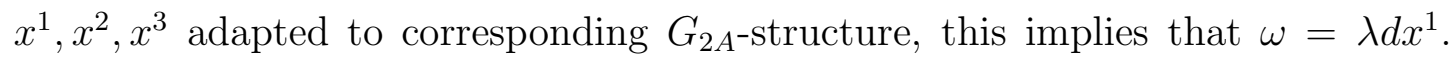
Since $d \omega=0$, after an appropriate change of coordinates we can assume that $\lambda=1$ and $\Omega=d x^{1} \wedge d x^{2} \wedge d x^{3}$.

$-\beta=0$, which is equivalent to case $\gamma=0$. A $G_{2 B}$-structure $P$ is given by a one-dimensional distribution $L$, a vector field $X$ and a volume form $\Omega$.

Proposition 6.12 $P$ is integrable if and only if

$$
\mathcal{L}_{X} L \subset L \text { and } \mathcal{L}_{X} \Omega=0 \text {. }
$$

\section{The group $2 \mathrm{C}$}

The group $G_{2 C}$ is the isotropy group of the vectors $e_{2}, e_{3}$ and the tensor $w$. Thus a $G_{2 C^{-}}$ structure $P$ is given by two vector fields $X_{1}, X_{2}$ which are linearly independent, and a volume form $\Omega$. We notice that $P$ can be alternatively described by $X_{1}, X_{2}$ and a one-form $\omega$ such that $\omega\left(X_{i}\right)=0, i=1,2$. In fact, given $\Omega$ we put $\omega=\iota_{X_{1}} \iota_{X_{2}} \Omega$, and given $\omega$, we define $\Omega$ by $\Omega\left(X_{1}, X_{2}, Z\right)=\omega(Z)$ for all vector field $Z$. 
Proposition 6.13 The following statements are equivalent:

(i) $P$ is integrable

(ii) $\left[X_{1}, X_{2}\right]=0$ and $\mathcal{L}_{X_{1}} \Omega=\mathcal{L}_{X_{2}} \Omega=0$.

(iii) $\left[X_{1}, X_{2}\right]=0$ and $d \omega=0$.

\section{The group 3A}

The group $G_{3 A}$ is the isotropy group of the subspaces $\left\langle e_{1}, e_{2}\right\rangle,\left\langle e_{2}, e_{3}\right\rangle$ and the tensor $w$. Therefore a $G_{3 A^{-}}$-structure $P$ is given by two distributions of dimension two $D_{1}, D_{2}$ and a volume form $\Omega$.

Proposition 6.14 $P$ is integrable if and only if $D_{1}$ and $D_{2}$ are both involutives.

Proof: Assume that $D_{1}$ and $D_{2}$ are both involutive. Then, by applying Proposition 6.1 to $L=D_{1} \cap D_{2} \subset D_{1}$ and $\Omega$, we obtain local coordinates $\left(y^{1}, y^{2}, y^{3}\right)$ such that $D_{1}=\left\langle\frac{\partial}{\partial y^{1}}, \frac{\partial}{\partial y^{2}}\right\rangle$ and $D_{1} \cap D_{2}=\left\langle\frac{\partial}{\partial y^{3}}\right\rangle$. So, $D_{2}=\left\langle\frac{\partial}{\partial y^{3}}, \frac{\partial}{\partial y^{2}}+a \frac{\partial}{\partial y^{1}}\right\rangle$, for some function $a$. Since $D_{2}$ is involutive, we deduce that $\frac{\partial a}{\partial y^{3}}=0$. It is clear that

$$
\lambda \frac{\partial}{\partial y^{1}}+\mu \frac{\partial}{\partial y^{3}}, \frac{\partial}{\partial y^{3}}, \frac{\partial}{\partial y^{2}}+a \frac{\partial}{\partial y^{1}}
$$

is an adapted local frame for all functions $\lambda$ and $\mu, \lambda \neq 0$. Now, the equation

$$
\left[\lambda \frac{\partial}{\partial y^{1}}+\mu \frac{\partial}{\partial y^{3}}, \frac{\partial}{\partial y^{2}}+a \frac{\partial}{\partial y^{1}}\right]=0
$$

is equivalent to the following system of PDE's:

$$
\left(\frac{\partial}{\partial y^{2}}+a \frac{\partial}{\partial y^{1}}\right) \lambda=-\frac{\partial a}{\partial y^{1}} \lambda,\left(\frac{\partial}{\partial y^{2}}+a \frac{\partial}{\partial y^{1}}\right) \mu=0 .
$$

In addition, we have that $\left[\frac{\partial}{\partial y^{3}}, \frac{\partial}{\partial y^{2}}+a \frac{\partial}{\partial y^{1}}\right]=0$ if and only if $\lambda$ and $\mu$ do not depend on $y^{3}$. Thus (9) have solution which are independent of $y^{3}$ if and only if $a$ does not depend on $y^{3}$. In such case, the other two brackets

$$
\left[\frac{\partial}{\partial y^{3}}, \lambda \frac{\partial}{\partial y^{1}}+\mu \frac{\partial}{\partial y^{3}}\right] \text { and }\left[\frac{\partial}{\partial y^{3}}, \frac{\partial}{\partial y^{2}}+a \frac{\partial}{\partial y^{1}}\right]
$$


also vanish and we conclude. The converse is trivial.

\section{The group 3B}

We only consider the algebraic case. This case is similar to the case $2 \mathrm{~B}$ but now the tensor fields are transverse to the one-dimensional distribution $L$ instead of tangent to the twodimensional distribution $D$. Concerning the integrability we obtain the following result.

Proposition 6.15 $\bar{P}$ is integrable if and only if it is projectable, i.e. the coefficients of the transverse tensor field $T$ in a system of coordinates $\left(x^{1}, x^{2}, x^{3}\right)$ adapted to the foliation induced by $L$ do not depend on the tangent coordinate $x^{3}$. Assume that $\bar{P}$ is integrable with adapted local coordinates $\left(x^{1}, x^{2}, x^{3}\right)$. Then $P$ is integrable if and only if $\mathcal{L}_{\frac{\partial}{\partial x^{1}}} \Omega=\mathcal{L}_{\frac{\partial}{\partial x^{2}}} \Omega=\mathcal{L}_{\frac{\partial}{\partial x^{3}}} \Omega=0$.

Proof: The last assertion is a consequence of Proposition 3.15, Remark 3.16 and the following computation of $\overline{\mathfrak{g}}^{(1)}$ : If $\tau=\left(\tau_{i}\right)_{i=1}^{3}$ with $\tau_{i}=\left(\begin{array}{ccc}\alpha a_{i} & b_{i} & c_{i} \\ 0 & \beta a_{i} & 0 \\ 0 & 0 & \gamma a_{i}\end{array}\right) \in \overline{\mathfrak{g}}$, then

\begin{tabular}{c|c|c|c|} 
& $\tau_{12}^{k}-\tau_{21}^{k}$ & $\tau_{13}^{k}-\tau_{31}^{k}$ & $\tau_{23}^{k}-\tau_{32}^{k}$ \\
\hline$k=1$ & $b_{1}-\alpha a_{2}$ & $c_{1}-\alpha a_{3}$ & $c_{2}-b_{3}$ \\
\hline$k=2$ & $\beta a_{1}-0$ & $0-0$ & $0-\beta a_{3}$ \\
\hline$k=3$ & $0-0$ & $\gamma a_{1}-0$ & $\gamma a_{2}-0$ \\
\hline
\end{tabular}

Consequently, if $\tau \in \overline{\mathfrak{g}}^{(1)}$ then $a_{1}=a_{2}=a_{3}=0$ and therefore $\operatorname{tr} \tau_{1}=\operatorname{tr} \tau_{2}=\operatorname{tr} \tau_{3}=0$.

\section{The group $3 \mathrm{C}$}

The group $G_{3 C}$ is the isotropy group of the covectors $e^{2}, e^{3}$ and the tensor $w$. Thus a

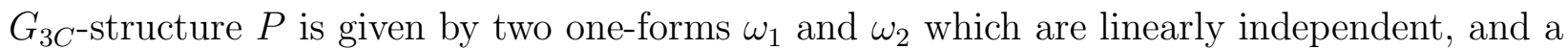
volume form $\Omega$. As in the case $2 \mathrm{C}$ we have an alternative description of $P$, giving $\omega_{1}, \omega_{2}$ and a vector field $X$ such that $\omega_{1}(X)=\omega_{2}(X)=0$.

Proposition 6.16 $P$ is integrable if and only if $\omega_{1}$ and $\omega_{2}$ are closed.

Proof: From Proposition 6.14 we conclude that there exist local coordinates $y^{1}, y^{2}, y^{3}$ such that $\omega_{i}=\lambda_{i} d y^{i}$ for $i=1,2$ and $\Omega=d y^{1} \wedge d y^{2} \wedge d y^{3}$. Since $d \omega_{i}=0$ then $\lambda_{i}$ only depends on $y^{i}$, after the change of coordinates given by $x^{i}=\int \lambda_{i}\left(y^{i}\right) d y^{i}, i=1,2$ and $x^{3}=\frac{y^{3}}{\lambda_{1} \lambda_{2}}$ we conclude. The converse is trivial. 


\section{The group $4 \mathrm{~A}$}

The group $G_{4 A}$ is the isotropy group of the subspaces $\left\langle e_{1}, e_{2}\right\rangle,\left\langle e_{2}\right\rangle,\left\langle e_{3}\right\rangle$ and the tensor $w$. Therefore a $G_{4 A}$-structure is given by one two-dimensional distribution $D$, two onedimensional distributions $L_{1}$ and $L_{2}$ such that $L_{1} \subset D$ and $L_{2} \cap D=0$, and a volume form $\Omega$.

Proposition 6.17 If $P$ is integrable then $D$ is involutive and the induced $G_{2 A}$-structure is integrable.

Remark 6.18 The converse does not hold. For instance take on $\mathbb{R}^{3}$ the $G_{4 A^{-}}$structure given by $L_{1}=\left\langle Y_{1}\right\rangle, L_{2}=\left\langle Y_{2}\right\rangle$ and $\Omega$ such that $\Omega\left(Y_{1}, Y_{2}, Y_{3}\right)=1$ where

$$
Y_{1}=\frac{\partial}{\partial y^{1}}, \quad Y_{2}=\frac{\partial}{\partial y^{2}}, \quad Y_{3}=y^{2} \frac{\partial}{\partial y^{1}}+\frac{\partial}{\partial y^{2}}+\frac{\partial}{\partial y^{3}}
$$

\section{The group 4B}

The group $G_{4 B}$ is the subgroup of $G_{2 B}$ which leaves the subspace $\left\langle e_{1}, e_{2}\right\rangle$ invariant. Thus, a $G_{4 B}$-structure consist of a $G_{2 B}$-structure $(L, T, \Omega)$ and a two-dimensional distribution $D^{\prime}$ which is complementary of $L$.

A necessary condition for the integrability of a $G_{4 B}$-structure is given by Propositions 6.10 . 6.12

\section{The group $4 \mathrm{C}$}

The group $G_{4 C}$ is the isotropy group of the vectors $e_{2}, e_{3}$ and the covector $e^{1}$. Therefore a $G_{4 C^{-}}$-structure $P$ is given by two vector fields which are linearly independent, and one-form $\omega$ such that $\omega\left(X_{1}\right)=0$ and $\omega\left(X_{2}\right)=0$.

Proposition 6.19 $P$ is integrable if and only if $\left[X_{1}, X_{2}\right]=0$ and $d \omega=0$.

\section{The group 5A}

The group $G_{5 A}$ is the isotropy group of the subspaces $\left\langle e_{1}\right\rangle,\left\langle e_{2}\right\rangle,\left\langle e_{3}\right\rangle$ and the tensor $w$. Alternatively, $G_{5 A}$ can be described as the isotropy group of a diagonalizable endomorphism $f$ with three distinct eigenvalues, and $w$. Then, a $G_{5 A}$-structure $P$ is given by three onedimensional distributions $L_{1}, L_{2}$ and $L_{3}$, and a volume form $\Omega$. Alternatively, $P$ can be described by a tensor field $h$ of type $(1,1)$ which is 0 -deformable to $f$, and the volume form $\Omega$. 
Remark 6.20 The Lie algebra $\mathfrak{g}$ is of finite type, indeed $\mathfrak{g}^{(1)}=0$, and then there is at most a free torsion $G_{5 A^{-}}$-connection $\nabla$. In fact, since the Nijenhuis tensor $N_{h}$ of $h$ vanishes one can constructs local $G_{5 A^{-}}$-connections without torsion and, since the uniqueness, they coincide on the overlappings.

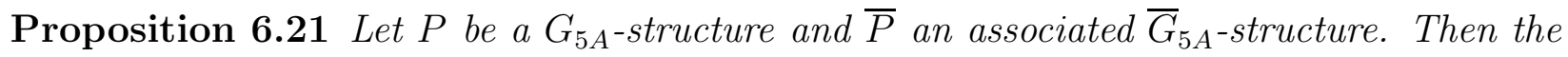
following statements are equivalent:

(i) $\bar{P}$ is integrable.

(ii) The distributions $L_{i} \oplus L_{j}$ are both involutive.

(iii) $N_{h}=0$.

Moreover, $P$ is integrable if and only if $N_{h}=0$ and the $G_{5 A^{-}}$-connection $\nabla$ has zero curvature.

\section{The group 5B}

We only consider the algebraic case, i.e., $\alpha, \beta$ and $\gamma$ are integer parameters. Reordering if it is necessary, we can assume that $\beta \geq 0$ and $\alpha \leq 0$. Then, the tensor

$$
t=\underbrace{e^{1} \otimes \cdots \otimes e^{1}}_{\beta} \otimes \underbrace{e_{2} \otimes \cdots \otimes e_{2}}_{-\alpha}
$$

is invariant by $G_{5 B}$. In fact, $G_{5 B}$ is the isotropy group of the subspaces $\left\langle e_{i}\right\rangle, i=1,2,3$, and the tensors $t$ and $w$. Therefore, a $G_{5 B}$-structure $P$ is given by three complementary one-dimensional distributions $L_{i}$, a tensor field $T$ which is 0-deformable to $t$, and a volume form $\Omega$. According to the precedent section we obtain that if $X_{1}, X_{2}, X_{3}$ is an adapted local frame to $P$ and we put $\left[X_{i}, X_{j}\right]=\gamma_{i j}^{k} X_{k}$, then the integrability of $P$ implies the following conditions:

$$
\gamma_{12}^{3}=0, \quad \gamma_{13}^{2}=0, \quad \gamma_{23}^{1}=0
$$

and

$$
\gamma \gamma_{12}^{1}+\alpha \gamma_{23}^{3}=0, \quad \gamma \gamma_{12}^{2}-\beta \gamma_{13}^{3}, \quad \beta \gamma_{13}^{1}-\alpha \gamma_{23}^{2}=0
$$

Remark 6.22 (i) Equations (10) imply that the distributions $L_{i} \oplus L_{j}$ are both involutive.

(ii) In this case $\partial_{\mathfrak{g}}$ is injective, therefore if Equations (10) and (11) hold, then there exist a unique torsionless $G_{5 B}$-connection $\nabla$. 
Proposition 6.23 $P$ is integrable if and only if $L_{i} \oplus L_{j}$ is involutive, Equations (11) hold for any local adapted frame to $P$, and the curvature of $\nabla$ vanishes.

Remark 6.24 The case $\alpha=0$ corresponds to a subgroup of $G_{19}$ which is conjugated with the special Lorentz group as we will see later. In this case, a $G_{5 B^{-}}$-structure is given by a Lorentzian metric $g$, its associated volume form, and a vector field $X$ such that $g(X, X)=1$. Then $P$ is integrable if and only if the Ricci tensor of $g$ is zero and $\nabla X=0$, where $\nabla$ is the Levi-Civita connection of $g$.

\section{The group 5C}

The group $G_{5 C}$ is the trivial group. Then a $G_{5 C}$-structure $P$ is just a linear parallelism $X_{1}, X_{2}, X_{3}$ on $B$.

Proposition 6.25 $P$ is integrable if and only if $\left[X_{1}, X_{2}\right]=\left[X_{1}, X_{3}\right]=\left[X_{2}, X_{3}\right]=0$, or, equivalently, if and only if the flat connection defined by the parallelism is symmetric.

\section{The group $6 \mathrm{~A}$}

The group $G_{6 A}$ is the isotropy group of the vector subspace $\left\langle e^{1} \otimes e^{1}+e^{2} \otimes e^{2}\right\rangle \subset T_{0}^{2} \mathbb{R}^{3}$ and $w$. Thus, giving a $G_{6 A^{-}}$-structure $P$ is equivalent to giving the projectivization of a symmetric covariant tensor field of order 2 and constant rank 2, and a volume form. But this is equivalent to give a one-dimensional distribution $L$ and a transverse almost complex structure $J$. We denote by $\bar{P}$ the $\bar{G}_{6 A^{-}}$structure obtained from $P$ without considering the volume form $\Omega$. Using the fact that all $G L(1, \mathbb{C})$-structure is integrable we obtain the following.

Proposition 6.26 $\bar{P}$ is integrable if and only if the transverse almost complex structure $J$ is projectable, i.e., the transverse tensor field $J$ does not depend on the tangent coordinates adapted to the foliation defined by $L$.

\section{The group $6 \mathrm{~B}$}

The only algebraic subgroup of type $6 \mathrm{~B}$ is obtained with $\alpha=0$. In this case, a $\bar{G}_{6 B}$-structure is given by a one-dimensional distribution $L$ and a Riemannian metric $g$ which is transverse to $L$. If in addition, we give a volume form $\Omega$ we obtain the corresponding $G_{6 B^{-}}$structure $P$. 
Proposition 6.27 $\bar{P}$ is integrable if and only if $g$ is projectable, i.e., $g$ is a transverse bundle like metric to the foliation defined by $L$.

\section{The group $7 \mathrm{~A}$}

The group $G_{7 A}$ is the isotropy group of the subspace $\left\langle e_{1} \otimes e_{1}+e_{2} \otimes e_{2}\right\rangle \subset T_{2}^{0} \mathbb{R}^{3}$ and the tensor $w$. Thus, a $G_{7 A}$-structure $P$ is given by the projectivization of a symmetric twocontravariant tensor field $g$ of rank 2, and a volume form $\Omega$. An alternative description of $\bar{P}$ consists of a two-dimensional distribution $D$ and a tangent almost complex structure on $D$. From Proposition 3.10 we obtain the following result.

Proposition 6.28 $\bar{P}$ is integrable if and only if $D$ is involutive.

\section{The group $7 \mathrm{~B}$}

The only algebraic subgroup of type $7 \mathrm{~B}$ is obtained by putting $\alpha=0$. This group $G_{7 B}$ is the isotropy group of the subspace $\left\langle e_{1}, e_{2}\right\rangle$, the tangent metric tensor $e^{1} \otimes e^{1}+e^{2} \otimes e^{2}$ and the tensor $w$. Then, a $G_{7 B}$-structure $P$ is given by a two-dimensional distribution $D$ with a tangent metric $g$ and a volume form $\Omega$.

Proposition 6.29 $\bar{P}$ is integrable if and only if $D$ is involutive and the scalar curvature of the metric defined on each leaf of the induced foliation vanishes. Assume that $\bar{P}$ is integrable with adapted local coordinates $\left(x^{1}, x^{2}, x^{3}\right)$. If $P$ is integrable then $\mathcal{L}_{\frac{\partial}{\partial x^{1}}} \Omega=\mathcal{L}_{\frac{\partial}{\partial x^{2}}} \Omega=0$.

Proof: The last assertion is a consequence of Remark 3.16 and the following calculation of $\overline{\mathfrak{g}}^{(1)}$ : If $\tau=\left(\tau_{i}\right)_{i=1}^{3}$ with $\tau_{i}=\left(\begin{array}{ccc}0 & a_{i} & b_{i} \\ -a_{i} & 0 & c_{i} \\ 0 & 0 & \beta_{i}\end{array}\right) \in \overline{\mathfrak{g}}$, then

\begin{tabular}{c|c|c|c|} 
& $\tau_{12}^{k}-\tau_{21}^{k}$ & $\tau_{13}^{k}-\tau_{31}^{k}$ & $\tau_{23}^{k}-\tau_{32}^{k}$ \\
\hline$k=1$ & $a_{1}-0$ & $b_{1}-0$ & $b_{2}-a_{3}$ \\
\hline$k=2$ & $0+a_{2}$ & $c_{1}+a_{3}$ & $c_{2}-0$ \\
\hline$k=3$ & $0-0$ & $\beta_{1}-0$ & $\beta_{2}-0$ \\
\hline
\end{tabular}

Consequently, if $\tau \in \overline{\mathfrak{g}}^{(1)}$ then $\beta_{1}=\beta_{2}=0$ and therefore $\operatorname{tr} \tau_{1}=\operatorname{tr} \tau_{2}=0$.

\section{The group $8 \mathrm{~A}$}


The group $G_{8 A}$ is the isotropy group of the endomorphism $f=e^{2} \otimes e_{1}-e^{1} \otimes e_{2}$ and the tensor $w$. Thus, a $\bar{G}_{8 A^{-}}$-structure is given by a tensor field $h$ of type $(1,1)$ which is 0 -deformable to $f$. We notice that $h^{3}+h=0$. Theses structures are called $f$-structures in the literature [35. If, in addition, we give a volume form $\Omega$ we obtain the corresponding $G_{8 A^{-}}$structure $P$.

Proposition 6.30 $\bar{P}$ is integrable if and only if $N_{h}=0$. In this case, there exist adapted local coordinates $x^{1}, x^{2}, x^{3}$ such that $\Omega=b\left(x^{1}, x^{2}, x^{3}\right) d x^{1} \wedge d x^{2} \wedge d x^{3}$, for some function $b$. Therefore, $P$ is integrable if and only if the following conditions hold:

$$
\frac{\partial^{2} \log b}{\partial x^{1} \partial x^{3}}=0, \quad \frac{\partial^{2} \log b}{\partial x^{2} \partial x^{3}}=0, \quad \frac{\partial^{2} \log b}{\partial\left(x^{1}\right)^{2}}+\frac{\partial^{2} \log b}{\partial\left(x^{2}\right)^{2}}=0 .
$$

Proof: The last assertion follows by applying the techniques described at the end of Section 3. By some calculations similar to the ones made in the proof of Proposition 6.29, we deduce that

$$
\overline{\mathfrak{g}}^{(1)}=\left\{\tau_{1}=\left(\begin{array}{ccc}
\alpha_{1} & \alpha_{2} & 0 \\
-\alpha_{2} & \alpha_{1} & 0 \\
0 & 0 & 0
\end{array}\right), \tau_{2}=\left(\begin{array}{ccc}
\alpha_{2} & -\alpha_{1} & 0 \\
\alpha_{1} & \alpha_{2} & 0 \\
0 & 0 & 0
\end{array}\right), \tau_{3}=\left(\begin{array}{ccc}
0 & 0 & 0 \\
0 & 0 & 0 \\
0 & 0 & \beta_{3}
\end{array}\right)\right\} .
$$

In order to obtain the integrability of $P$ we need to construct a tensor field $\tau: U \rightarrow \overline{\mathfrak{g}}^{(1)}$ such that $\frac{\partial \tau_{j}}{\partial x^{i}}-\frac{\partial \tau_{i}}{\partial x^{j}}+\left[\tau_{i}, \tau_{j}\right]=0$ and $\operatorname{tr} \tau_{i}=\frac{\partial \log b}{\partial x^{2}}$, where $\Omega=b\left(x^{1}, x^{2}, x^{3}\right) d x^{1} \wedge d x^{2} \wedge d x^{3}$. Taking into account that $\left[\tau_{i}, \tau_{j}\right]=0, \frac{\partial \log b}{\partial x^{i}}=\operatorname{tr} \tau_{i}=2 \alpha_{i}$ if $i=1,2$ and $\frac{\partial \log b}{\partial x^{3}}=\operatorname{tr} \tau_{3}=\beta_{3}$, the compatibility relations of the resulting system of PDE's can be expressed as (12).

\section{The group $8 \mathrm{~B}$}

The only algebraic subgroup of type $8 \mathrm{~B}$ is obtained when $\alpha=0$. In this case $G_{8 B}$ is the subgroup of $S O(3)$ that leaves invariant the vector $e_{3}$. Thus, a $G_{8 B}$-structure $P$ is given by a Riemannian metric $g$, the Riemannian volume form $\Omega$, and a vector field $X$ without zeros.

Proposition 6.31 $P$ is integrable if and only if the Ricci tensor of $g$ vanishes and $\nabla X=0$ where $\nabla$ is the Levi-Civita connection of $g$.

\section{The group 9}

The group $G_{9}$ is the special linear group $S l(3, \mathbb{R})$. Then a $G_{9}$-structure is given by a volume form $\Omega$. If we look in the proof of Proposition 6.1. we deduce the following result.

Proposition 6.32 Every $S l(3, \mathbb{R})$-structure is integrable. 


\section{The group 10}

The group $G_{10}$ is the isotropy group of the subspace $\left\langle e_{1}, e_{2}\right\rangle$ of $\mathbb{R}^{3}$ and the tensor $w$. Then a $G_{10}$-structure $P$ is given by a two-dimensional distribution $D$ and a volume form $\Omega$.

Proposition 6.33 $P$ is integrable if and only if the distribution $D$ is involutive.

\section{The group 11}

The group $G_{11}$ is the isotropy group of the subspace $\left\langle e_{3}\right\rangle$ of $\mathbb{R}^{3}$ and the tensor $w$. Thus, a $G_{11}$-structure $P$ is given by a one-dimensional distribution $L$ and a volume form $\Omega$.

Proposition 6.34 $P$ is always integrable.

\section{The group 12}

The group $G_{12}$ is the isotropy group of the covector $e^{3}$ and the tensor $w$. Therefore, a $G_{12}$-structure $P$ is given by a one-form $\omega$ without zeros and a volume form $\Omega$.

Proposition 6.35 $P$ is integrable if and only if $d \omega=0$.

\section{The group 13}

The group $G_{13}$ is the isotropy group of the vector $e_{3}$ and the tensor $w$. Then, a $G_{13}$-structure $P$ is given by a vector field $X$ without zeros and a volume form $\Omega$.

Proposition 6.36 $P$ is integrable if and only if $\mathcal{L}_{X} \Omega=0$.

\section{The group 14}

The group $G_{14}$ is the isotropy group of the subspaces $\left\langle e_{1}, e_{2}\right\rangle,\left\langle e_{3}\right\rangle$ of $\mathbb{R}^{3}$ and the tensor $w$. Alternatively, it is the isotropy group of the endomorphism $e^{1} \otimes e_{1}$ and the tensor $w$. Thus, a $G_{14}$-structure $P$ is given by a two-dimensional distribution $D$, a complementary onedimensional distribution $L$, and a volume form $\Omega$. Alternatively, it is given by a tensor field $h$ of type $(1,1)$ such that $h^{3}-h^{2}=0$, together with the form $\Omega$. Let $\bar{P}$ be the $\bar{G}_{14}$-estructure obtained from $P$ without considering the volume form $\Omega$. 
Proposition 6.37 $\bar{P}$ is integrable if and only if $N_{h}=0$.

\section{The group 15}

The group $G_{15}$ is the isotropy group of the tensors $u=e^{1} \wedge e^{2}$ and $v=e^{3}$. We notice that $w=u \wedge v$. Therefore a $G_{15}$-structure $P$ is given by a 2 -form $\eta$ and a 1 -form $\omega$ such that $\eta \wedge \omega \neq 0$ everywhere. Theses structures are called almost-cosymplectic [3].

Proposition 6.38 $P$ is integrable if and only if $d \eta=0$ and $d \omega=0$.

Proof: It is just the statement of Darboux's Theorem (see [3]).

\section{The group 16}

The group $G_{16}$ is the special orthogonal group $S O(3)$. Then a $G_{16}$-structure $P$ is given by a Riemannian metric $g$ on $B$ and the Riemannian volume form $\Omega$.

Proposition 6.39 $P$ is integrable if and only if the curvature of the Levi-Civita connection of $g$ vanishes. This condition is equivalent to the vanishing of the Ricci tensor of $g$.

\section{The group 19}

The group $G_{19}$ is the isotropy group of the tensors

$$
s=e^{2} \otimes e^{3}+e^{3} \otimes e^{2}-e^{1} \otimes e^{1}
$$

and $w$. We notice that $s$ is in the same orbit that $-e^{1} \otimes e^{1}-e^{2} \otimes e^{2}+e^{3} \otimes e^{3}$, which is the model for the Lorentzian metrics, thus the group $G_{19}$ is conjugated with the special group $S O(2,1)$. Since we are discussing subgroups modulo conjugation we can consider a $G_{19}$-structure as given by a Lorentz metric $g$ and its volume form.

Proposition 6.40 As in the Riemannian case, $P$ is integrable if and only if the Ricci tensor of $g$ is zero.

\section{The group 20}

The group $G_{20}$ is the isotropy group of the subspace of $T_{0}^{4} \mathbb{R}^{3}$ generated by

$$
t=\left(e^{2} \otimes e^{3}+e^{3} \otimes e^{2}-e^{1} \otimes e^{1}\right) \otimes e^{3} \otimes e^{3}-e^{3} \otimes e^{3} \otimes\left(e^{2} \otimes e^{3}+e^{3} \otimes e^{2}-e^{1} \otimes e^{1}\right)
$$


and the tensor $w$. Therefore a $G_{20}$-structure $P$ is given by the projectivization of a tensor field $T$ which is 0 -deformable to $t$, and a volume form $\Omega$. We can use the conditions in Section 3, however we did not find any nice geometrical interpretation.

\section{The group 23}

The group $G_{23}$ is the subgroup of $G_{19}$ which leaves the subspace $\left\langle e_{2}\right\rangle$ invariant. Via conjugation of $G_{19}$ with the special group $S O(2,1), e_{2}$ becomes a vector in the light cone. Thus, a $G_{23}$-structure $P$ is given by a Lorentzian metric $g$, its volume form $\Omega$ and a one-dimensional distribution $L$ contained in the light cone of $g$.

Proposition 6.41 $P$ is integrable if and only if the Ricci tensor of $g$ vanishes and $\nabla L \subset L$, where $\nabla$ is the generalized Levi-Civita connection of $g$.

\section{The group 24}

The group $G_{24}$ is conjugated with the isotropy group of the tensor $w$ and the endomorphism $u=e^{2} \otimes e_{1}+e^{3} \otimes e_{2}$, with minimum polynomial $u^{3}=0$. Then, a $\bar{G}_{24}$-structure $\bar{P}$ is given by a tensor field $h$ which is 0 -deformable to $u$. If, in addition, we give a volume form $\Omega$ then we obtain the corresponding $G_{24}$-structure $P$.

Proposition 6.42 $\bar{P}$ is integrable if and only if $N_{h}=0$. Assuming that $\bar{P}$ is integrable, then we deduce that $P$ is integrable if and only if for an arbitrary system of coordinates $x^{1}, x^{2}, x^{3}$ adapted to $\bar{P}$ the following conditions hold:

$$
\mathcal{L}_{\frac{\partial}{\partial x^{1}}} \Omega=0, \quad \mathcal{L}_{\frac{\partial}{\partial x^{2}}} \Omega=0
$$

Proof: Using the conjugation between $\bar{G}_{24}$ and $\bar{G}=G_{u}$ we can characterize the integrability of $P$ in terms of $\overline{\mathfrak{g}}=\left\{\left(\begin{array}{lll}a & b & c \\ 0 & a & b \\ 0 & 0 & a\end{array}\right) \mid a, b, c \in \mathbb{R}\right\}$. First of all, we compute $\overline{\mathfrak{g}}^{(1)}$ by means of the following table

\begin{tabular}{c|c|c|c|} 
& $\tau_{12}^{k}-\tau_{21}^{k}$ & $\tau_{13}^{k}-\tau_{31}^{k}$ & $\tau_{23}^{k}-\tau_{32}^{k}$ \\
\hline$k=1$ & $b_{1}-a_{2}$ & $c_{1}-a_{3}$ & $c_{2}-b_{3}$ \\
\hline$k=2$ & $a_{1}-0$ & $b_{1}-0$ & $b_{2}-a_{3}$ \\
\hline$k=3$ & $0-0$ & $a_{1}-0$ & $a_{2}-0$ \\
\hline
\end{tabular}


obtaining that

$$
\overline{\mathfrak{g}}^{(1)}=\left\{\tau_{1}=\left(\begin{array}{ccc}
0 & 0 & a_{3} \\
0 & 0 & 0 \\
0 & 0 & 0
\end{array}\right), \tau_{2}=\left(\begin{array}{ccc}
0 & a_{3} & b_{3} \\
0 & 0 & a_{3} \\
0 & 0 & 0
\end{array}\right), \tau_{1}=\left(\begin{array}{ccc}
a_{3} & b_{3} & c_{3} \\
0 & a_{3} & b_{3} \\
0 & 0 & a_{3}
\end{array}\right)\right\} .
$$

The integrability of $P$ is equivalent to the existence of a tensor field $\tau: U \rightarrow \overline{\mathfrak{g}}^{(1)}$ verifying the system of PDE's $\frac{\partial \tau_{j}}{\partial x^{i}}-\frac{\partial \tau_{i}}{\partial x^{j}}+\left[\tau_{i}, \tau_{j}\right]=0$, jointly to the equations $\operatorname{tr} \tau_{i}=\frac{\partial \log b}{\partial x^{i}}$, being $\Omega=b\left(x^{1}, x^{2}, x^{3}\right) d x^{1} \wedge d x^{2} \wedge d x^{3}$. Taking into account that $\left[\tau_{i}, \tau_{j}\right]=0$, it is easy to check that the precedent system of PDE's has always solution provides that $a_{3}=\frac{1}{3} \frac{\partial \log b}{\partial x^{3}}$ and the function $b\left(x^{1}, x^{2}, x^{3}\right)$ does not depend on $x^{1}$ nor $x^{2}$, what is equivalent to (13).

\section{The group 26}

The group $G_{26}$ is another subgroup of $G_{19}$. Under the same identifications as in that case, and by similar computations we conclude that giving a $G_{26}$-structure $P$ is the same that giving a Lorentzian metric $g$, its volume form $\Omega$, and a isotropic vector field $X$ without zeros (i.e., such that $g(X, X)=0$ ).

Proposition 6.43 $P$ is integrable if and only if the Ricci tensor of $g$ vanishes and $\nabla X=0$, where $\nabla$ is the generalized Levi-Civita connection of $g$.

\section{Chevalley's Theorem}

As we have shown, a $G$-structure defined by a 0-deformable tensor field is a reduction of the frame bundle $\mathcal{F} M$ to an algebraic subgroup $G$ of $G l(n, \mathbb{R})$. We can ask for a converse: given a $G$-structure with $G$ an algebraic subgroup of $G l(n, \mathbb{R})$, there exist a 0-deformable tensor field defining it?

An approach to this question is given by a Theorem of Chevalley whose proof we sketch below. Before stating it, let us introduce some notions. Let $V$ be a vector space. A construction over $V$ is a vector space obtained from $V$ by iterating the operations $*, \oplus, \otimes, S^{m}$ and $\bigwedge^{m}$. If $g \in G L(V)$ then $g$ acts in a natural way on each construction over $V$ following the rules:

(i) if $v \in V$ then $\rho(g)(v)=g v$ is the standard action,

(ii) if $\omega \in V^{*}$ then $\rho(g)(\omega): v \mapsto \omega\left(g^{-1} v\right)$,

(iii) $\rho(g)(a \otimes b)=\rho(g)(a) \otimes \rho(g)(b)$, 
(iv) $\rho(g)(a \oplus b)=\rho(g)(a) \oplus \rho(g)(b)$.

In other words, $\rho$ defines the (faithful) tensorial representation $\rho: G L(V) \rightarrow G L\left(T_{V}\right)$, where $T_{V}=\bigoplus_{r, s \geq 0} V^{r, s}\left(\right.$ with $\left.V^{r, s}=V^{\otimes r} \otimes\left(V^{*}\right)^{\otimes s}\right)$ is the whole tensor algebra over $V$.

Remark 7.1 Any construction $W$ over $V$ is a direct sum of finite dimensional subspaces of the whole tensor algebra $T_{V}$. Therefore, if $M$ is a manifold of dimension $n=\operatorname{dim} V$, the associated vector bundle $(\mathcal{F} M \times W) / G l(n, \mathbb{R})$ is a direct sum of tensor bundles over $M$. On the other hand, if $u \in W$ and $G=\{g \in G l(n, \mathbb{R}) \mid \rho(g) u=u\}$ (respectively, $G=\{g \in$ $G l(n, \mathbb{R}) \mid \exists \lambda \neq 0, \rho(g) u=\lambda u\})$ then, according to Theorem 2.2 , any $G$-structure is given by some $W$-tensor (respectively $\mathbb{P} W$-tensor) which is 0 -deformable to $u \in W$ (respectively, $[u] \in \mathbb{P} W)$.

It is easy to prove that if $\left\{V_{i}\right\}_{i \in I}$ is a family of constructions, and for every $i \in I,\left\{W_{i j}\right\}_{j \in J_{i}}$ is a family of subspaces of $V_{i}$, then

$$
H=\left\{g \in G L(V) \mid g\left(W_{i j}\right) \subset W_{i j}, \forall i \in I, \forall j \in J_{i}\right\}
$$

is an algebraic subgroup of $G L(V)$. The following result is a weak converse.

Theorem 7.2 (Chevalley) (see [2, 30]) Let $H$ be an algebraic subgroup of $G L(V)$. Then there exist a finite-dimensional vector space $W$, a faithful representation $\alpha: G L(V) \rightarrow$ $G L(W)$ and a subspace $W_{0} \subset W$ such that

$$
H=\left\{g \in G L(V) \mid \alpha(g)\left(W_{0}\right) \subset W_{0}\right\}
$$

In fact, $W_{0}$ and $W$ can be chosen such that $\operatorname{dim} W_{0}=1$.

Sketch of the proof (from [2, 5]): For the sake of shortness we will write $G$ instead of $G L(V)$ and we denote by $K[G]$ the ring of regular functions over $G$ with values in the ground field $K\left(K=\mathbb{R}\right.$ in what follows), i.e. $K[G]=K\left[x_{i j}, 1 \leq i, j \leq n\right]\left[\Delta^{-1}\right]$ with $\Delta=\operatorname{det}\left(x_{i j}\right)$ and $n=\operatorname{dim} V$.

Since $G$ acts on itself by left translations, it induces a linear action on $K[G]$ by pull-back. More precisely, if $g \in G$ and $f \in K[G]$ then $g_{*} f:=\left(g^{-1}\right)^{*} f:\left(x_{i j}\right) \mapsto f\left(g^{-1} \cdot\left(x_{i j}\right)\right)$. The map $g \mapsto g_{*}$ defines a faithful representation $\alpha: G \rightarrow G L(K[G])$. The space $W$ stated in the theorem will be a suitable subspace of $K[G]$ with the restricted representation $\alpha: G \rightarrow G L(W)$.

Since $H \subset G$ is algebraic, we can consider the ideal $I(H) \subset K[G]$ defining $H$, i.e. $I(H)=$ $\{f \in K[G] \mid f(h)=0$ for all $h \in H\}$. Using that $K[G]$ is noetherian, we can find a finite 
dimensional subspace $W \subset K[G]$ containing a system of generators of $I(H)$. Then, we define $W_{0}$ as $W \cap I(H)$ and we have

$$
H=\left\{g \in G \mid g^{-1} \cdot H \subset H\right\}=\{g \in G \mid \alpha(g)(I(H)) \subset I(H)\}=\left\{g \in G \mid \alpha(g)\left(W_{0}\right) \subset W_{0}\right\} .
$$

Finally, if $k=\operatorname{dim} W_{0}>1$ then we can take $\alpha^{\prime}=\bigwedge^{k} \alpha, W^{\prime}=\bigwedge^{k} W$ and $W_{0}^{\prime}=\bigwedge^{k} W_{0}$ which is one-dimensional.

Remark 7.3 Denoting by $G=G L(V)$ and by $E=E n d(V)=V \otimes V^{*}$, notice that $K[G]$ contains $K\left[x_{i j}\right]=K[E] \cong S\left(E^{*}\right)=\bigoplus_{s \geq 0}\left(E^{*}\right)^{\otimes s} \hookrightarrow T_{V}$. On the other hand, from the proof we can choose $W \subset K[E]$. Thus, we can take $W \subset T_{V}$, but the representation $\alpha: G L(V) \rightarrow$ $G L(W)$ is not the restriction of the tensorial representation $\rho: G L(V) \rightarrow G L\left(T_{V}\right)$ considered above.

Although in some references [7, 29] it seems that Chevalley's theorem holds for $\alpha=\rho$, we have not found a proof of this. Therefore, we prefer do not use this stronger version and, consequently, we show, in the same spirit, a slightly different result:

Theorem 7.4 Let $H$ be an algebraic subgroup of $G L(V)$. Then there exists a finite dimensional subspace $W \subset T_{V}$ such that the normalizer $\mathcal{N}(H)=\left\{g \in G L(V) \mid g^{-1} H g=H\right\}$ of $H$ verifies:

$$
\mathcal{N}(H)=\{g \in G L(V) \mid \rho(g)(W) \subset W\},
$$

where $\rho: G L(V) \rightarrow G L\left(T_{V}\right)$ denotes the tensorial representation. Moreover, we can take $W$ one-dimensional.

Proof: Consider the adjoint action of $G$ onto itself and the induced representation $\rho^{\prime}: G \rightarrow$ $G L(K[G])$ given by $\rho^{\prime}(g)(f):\left(x_{i j}\right) \mapsto f\left(g^{-1} \cdot\left(x_{i j}\right) \cdot g\right)$ for any $f \in K[G]$. A straightforward calculation shows that, under the identifications made in Remark 7.3, $\rho^{\prime}$ coincides with the tensorial representation $\rho: G \rightarrow G L\left(T_{V}\right)$.

Now, we proceed as above by considering the ideal $I(H)$ of $K[G]$ defining the algebraic subgroup $H \subset G$. Again, since $K[G]$ is noetherian there is a finite dimensional subspace $W$ of $K[E] \subset K[G]$ which contains a system of generators of $I(H)$. Identifying $W$ as a subspace of $T_{V}$, we have

$$
\begin{aligned}
& \{g \in G \mid \rho(g)(W) \subset W\}=\left\{g \in G \mid \rho^{\prime}(g)(I(H)) \subset I(H)\right\} \\
= & \left\{g \in G \mid \rho^{\prime}(g)(f) \in I(H), \forall f \in I(H)\right\}=\left\{g \in G \mid \rho^{\prime}(g)(f)(h)=0, \forall f \in I(H), \forall h \in H\right\} \\
= & \left\{g \in G \mid f\left(g^{-1} h g\right)=0, \forall f \in I(H), \forall h \in H\right\}=\left\{g \in G \mid g^{-1} h g \in H, \forall h \in H\right\} \\
= & \mathcal{N}(H) .
\end{aligned}
$$

which proves the result. The last assertion follows taking a suitable exterior power of $W$. 
Theorem 7.5 Let $G$ be an algebraic subgroup of $G l(n, \mathbb{R})$. Then, any $\mathcal{N}(G)$-structure can be given by the projectivization of an inhomogeneous tensor field $t=\sum_{i=1}^{m} t_{i}$, where each $t_{i}$ is a 0-deformable tensor field on $M$ of type $\left(r_{i}, s_{i}\right)$.

Remark 7.6 In [10] the authors explore the possibility of the existence of non-uniform materials which could enjoy, however, some kind of homogeneity. They introduce the notion of unisymmetric materials as follows.

Definition 7.7 A material body is said to be unisymmetric if the material symmetry groups of its points in one (and, therefore, in every) configuration are pairwise conjugate.

Functionally graded materials (FGM for short), important for their industrial applications, are of this type.

Let $X_{1}$ and $X_{2}$ be two points of a unisymmetric body $B$, and let $A: T_{X_{1}} B \longrightarrow T_{X_{2}} B$ be a symmetry isomorphism such that $G_{2}=A G_{1} A^{-1}$, where $G_{1}$ and $G_{2}$ are the material symmetry groups at $X_{1}$ and $X_{2}$, respectively. Then, the family $\mathcal{A}_{12}$ of all possible symmetry isomorphisms between both points is

$$
\mathcal{A}_{12}=A \mathcal{N}\left(G_{1}\right)
$$

where $\mathcal{N}\left(G_{1}\right)$ is the normalizer of $G_{1}$ in $G l(n, \mathbb{R})$. If we proceed as in Section 5 for uniform materials, and choose a point $X_{0}$ and a particular linear frame $Z_{0}$ at $X_{0}$, we can transport the material symmetry group $G\left(X_{0}\right)$ at $X_{0}$ to $\mathbb{R}^{n}$ and obtain a subgroup $G$ of $G l(n, \mathbb{R})$. Using (14) we deduce that possible admissible references at $X_{0}$ is just $Z_{0} \mathcal{N}(G)$, where $\mathcal{N}(G)$ is the normalizer of $G$. This means that the geometric structure associated to a unisymmetric body is a $\mathcal{N}(G)$-structure. Accordingly to Theorem 7.4 this implies that, if $G$ is an algebraic subgroup of $G l(n, \mathbb{R})$ then the geometric $\mathcal{N}(G)$-structure is defined by the projectivization of a tensor. This fact probably deserves a more careful analysis to be done elsewhere.

\section{Acknowledgments}

This work has been supported through grants DGICYT (Spain), Projects PB94-0106, PB971257, BFM2001-2272 and NATO CRG 950833. D.M. acknowledges the support of the Consejo Superior de Investigaciones Científicas through a grant. We acknowledge to Profs. Marcel Nicolau and Agustí Reventós for their help during the preparation of this paper. M. de L. wish to acknowledge the warm hospitality of the Departament de Matemàtiques (Universitat Autònoma de Barcelona). 


\section{A. Connected Lie subgroups of the $S l(3, \mathbb{R})$}

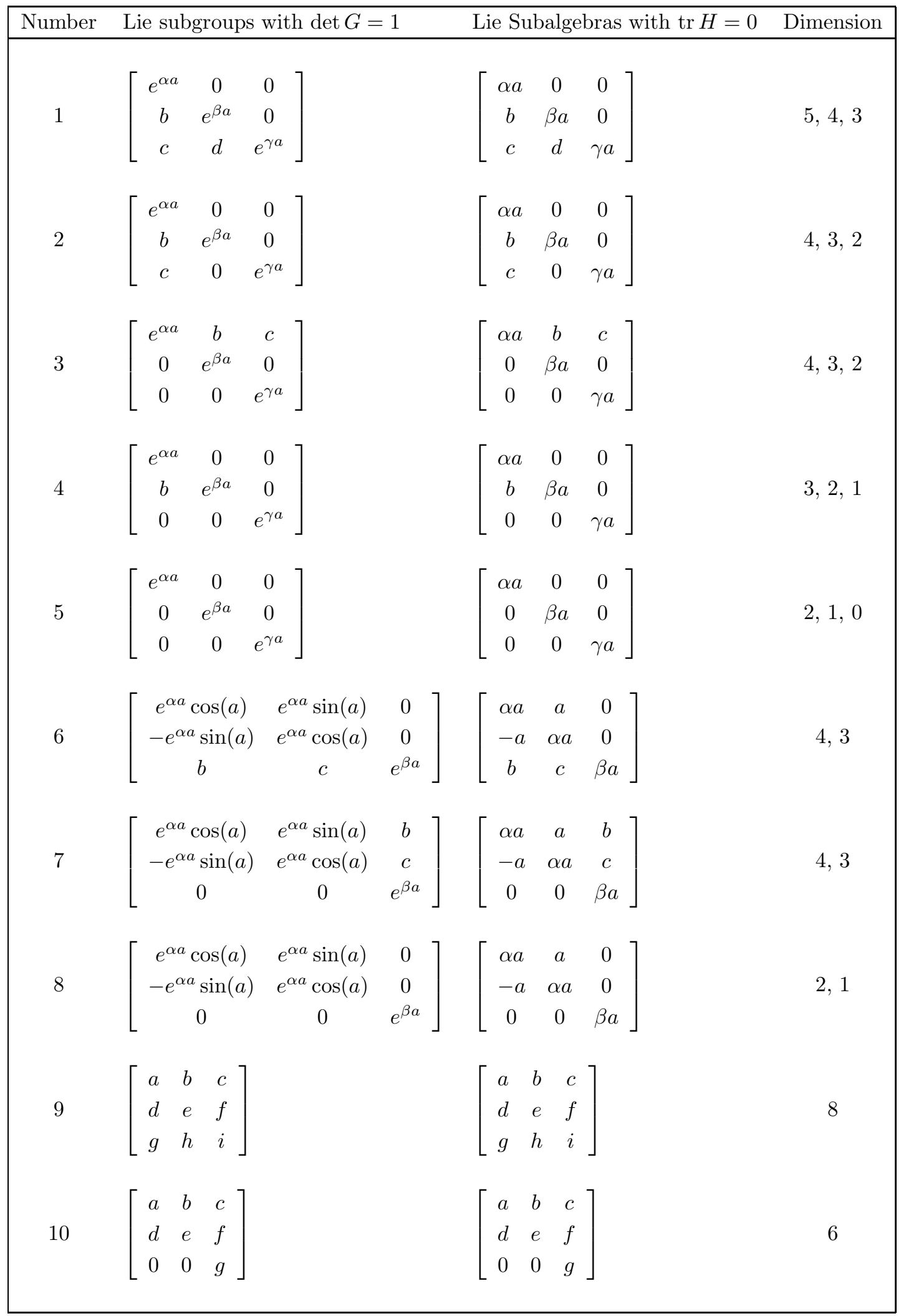




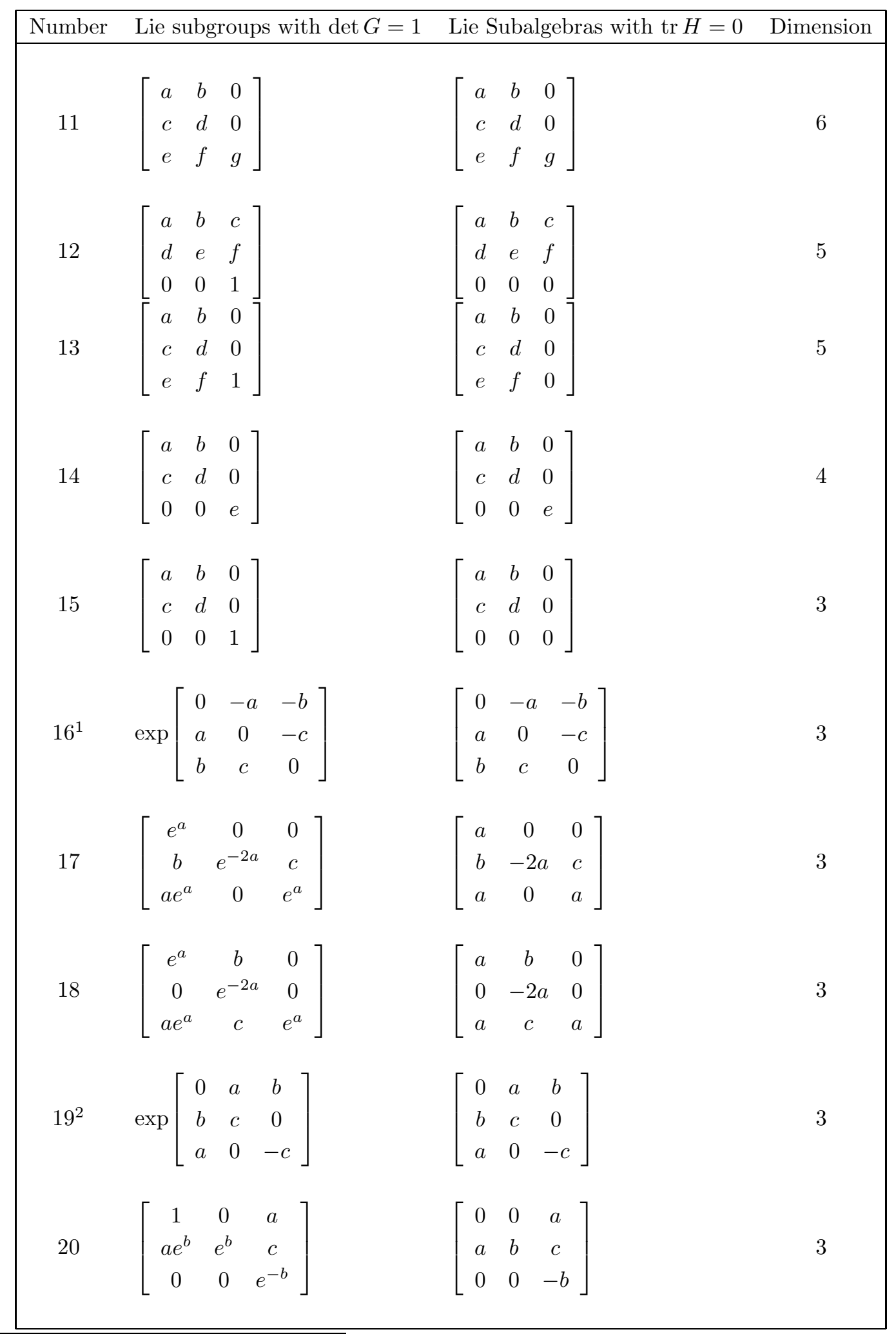

${ }^{1}$ The explicit form of this subgroup is very complicated but is just the special orthogonal group.

${ }^{2}$ This subgroup is conjugate to the Lorentz group. 


\begin{tabular}{|c|c|c|c|}
\hline Number & Lie subgroups with $\operatorname{det} G=1$ & Lie Subalgebras with $\operatorname{tr} H=0$ & Dimension \\
\hline 21 & {$\left[\begin{array}{ccc}e^{a} & 0 & 0 \\
b & e^{-2 a} & 0 \\
a e^{a} & 0 & e^{a}\end{array}\right]$} & {$\left[\begin{array}{ccc}a & 0 & 0 \\
b & -2 a & 0 \\
a & 0 & a\end{array}\right]$} & 2 \\
\hline 22 & {$\left[\begin{array}{ccc}e^{a} & 0 & 0 \\
0 & e^{-2 a} & 0 \\
a e^{a} & b & e^{a}\end{array}\right]$} & {$\left[\begin{array}{ccc}a & 0 & 0 \\
0 & -2 a & 0 \\
a & b & a\end{array}\right]$} & 2 \\
\hline 23 & {$\left[\begin{array}{ccc}1 & 0 & a \\
a e^{b} & e^{b} & \frac{1}{2} a^{2} e^{b} \\
0 & 0 & e^{-b}\end{array}\right]$} & {$\left[\begin{array}{ccc}0 & 0 & a \\
a & b & 0 \\
0 & 0 & -b\end{array}\right]$} & 2 \\
\hline 24 & {$\left[\begin{array}{lll}1 & a & 0 \\
0 & 1 & 0 \\
a & b & 1\end{array}\right]$} & {$\left[\begin{array}{lll}0 & a & 0 \\
0 & 0 & 0 \\
a & b & 0\end{array}\right]$} & 2 \\
\hline 25 & {$\left[\begin{array}{ccc}e^{a} & 0 & 0 \\
0 & e^{-2 a} & 0 \\
a e^{a} & 0 & e^{a}\end{array}\right]$} & {$\left[\begin{array}{ccc}a & 0 & 0 \\
0 & -2 a & 0 \\
a & 0 & a\end{array}\right]$} & 1 \\
\hline 26 & {$\left[\begin{array}{ccc}1 & a & 0 \\
0 & 1 & 0 \\
a & \frac{1}{2} a^{2} & 1\end{array}\right]$} & $\left.\begin{array}{lll}0 & a & 0 \\
0 & 0 & 0 \\
a & 0 & 0\end{array}\right]$ & 1 \\
\hline
\end{tabular}




\section{References}

[1] D. Bernard: Sur la géométrie différentielle des $G$-structures, Ann. Inst. Fourier 10 (1960), 151-270.

[2] D. Bertrand: Groupes algébriques linéaires et théorie de Galois différentielle, Cours de 3ème cycle, 1985-86, Université Paris VI.

[3] D.E. Blair: Contact manifolds in Riemannian geometry, Springer-Verlag, Berlin, 1976.

[4] F. Bloom: Modern Differential Geometric Techniques in the Theory of Continuous Distributions of Dislocations, Lecture Notes in Math. 733, Springer, Berlin, 1979.

[5] A. Borel: Linear Algebraic Groups, Graduate Texts in Mathematics 126, Springer, New-York, 1991.

[6] S.S. Chern: The geometry of G-structures, Bull. Amer. Math. Soc. 72 (1966), 167-219.

[7] C. Chevalley: La théorie des groupes algébriques, Proceedings of the International Congress of Mathematicians, 14-21 August 1958 (Edimburg)

[8] L.A. Cordero, C.T.J. Dodson, M. de León: Differential Geometry of Frame Bundles, Mathematics and Its Applications, Kluwer, Dordrecht, 1989.

[9] M. Elzanowski, M. Epstein, J. Sniatycki: G-structures and material homogeneity, Journal of Elasticity 23 (1990), 167-180.

[10] M. Epstein, M. de León: Homogeneity without uniformity: towards a mathematical theory of functionally graded materials, Int. J. of Solids Structures 37 (2000), 75777591.

[11] A. Fujimoto: Theory of G-structures, Publications of the Study Group of Geometry, Vol. I. Tokyo, 1972.

[12] W. Greub, S.Halperin, R. Vanstone: Connections, Curvature, Cohomology, Vol I. Acad. Press, New York-London, 1972.

[13] E.T. Kobayashi: A remark on the existence of $G$-structure, Proc. Amer. Math. Soc. 16 (1959), 300-309.

[14] E.T. Kobayashi: A remark on the Nijenhuis tensor, Pacific Journal of Math. 12 (1962), 963-977. 
[15] S. Kobayashi, K. Nomizu: Foundations of Differential Geometry, vol. I, Interscience Publishers, New York, 1963.

[16] S. Kobayashi: Transformations Groups in Differential Geometry, Springer, Berlin-New York, 1972.

[17] J. Lehmann-Lejeune: Sur l'integrabilite de certaines G-structures, C.R. Acad. Sc. Paris 258 (1964), 5326-5329.

[18] M. de León, M. Epstein: On the integrability of second order G-structures with applications to continuous theories of dislocations. Reports on Mathematical Physics 33 (3) (1993), 419-436.

[19] M. de León, M. Epstein: Corps matériels de degré supérieur. Comptes Rendus Acad. Sc. Paris 319, Série I, (1994), 615-620.

[20] M. de León, M. Epstein: Homogeneity conditions for generalized Cosserat media. Journal of Elasticity 43 (1996), 189-201.

[21] M. de León, M. Epstein: On uniformity of shells. Int. J. of Solids Structures 3517 (1998), 2173-2182.

[22] M. de León, M. Epstein: Geometrical Theory of Uniform Cosserat Media. Journal of Geometry and Physics 26 (1998), 127-170. (Con M. Epstein).

[23] S. Lie, F. Engel: Theorie der transformationsgruppen, Vol 3. Leipzig. Teubner 1893.

[24] K. Mackenzie: Lie groupoids and Lie algebroids in Differential Geometry, London Mathematical Society Lecture Note Series 124, Cambridge Univ. Press, Cambridge, 1987.

[25] J.E. Marsden, T.J.R. Hughes: Mathematical Foundations of Elasticity, Prentice Hall, New Jersey, 1983.

[26] G.A. Maugin: Material Inhomogeneities in Elasticity, London, Chapman \& Hall, 1993.

[27] W. Noll: Materially Uniform Simple Bodies with Inhomogeneities, Arch. Rational Mech. Anal. 27, (1967), 1-32.

[28] T. Nôno: On the symetry groups of simple materials: An application of the theory of Lie groups, Journal Math. Anal. Appl. 24 (1968), 110-135.

[29] A.L. Onishchik, E.B. Vinberg: Lie Groups and Algebraic Groups, Springer-Verlag, Berlin-Heidelberg, 1990. 
[30] J.P. Ramis, J. Martinet, Théorie de Galois Différentielle et Resommation, Part 2 of Computer Algebra and Differential Equations, Academic Press, 1990.

[31] C. Truesdell, R.A. Toupin: Principles of Classical Mechanics and Field Theory, Handbuch der Physik, Vol. III/1, Berlin-New York, Springer, 1960.

[32] C. Truesdell, W. Noll: The Non-Linear Field Theories of Mechanics, Handbuch der Physik, Vol. III/3, Berlin-New York, Springer, 1965.

[33] C.C. Wang, C. Truesdell: Introduction to rational elasticity, Noordhoff International Publishing, Leyden, 1973.

[34] C.C. Wang: On the Geometric Structures of Simple Bodies, a Mathematical Foundation for the Theory of Continuous Distributions of Dislocations, Arch. Rational Mech. Anal. 27, (1967), 33-94.

[35] K. Yano, M. Kon: Structures on manifolds, World Scientific, Singapore, 1984.

DAVID MARÍN

Departament de Matemàtiques, Universitat Autònoma de Barcelona, 08193 Bellaterra, Barcelona, SPAIN

e-mail: davidmp@mat.uab.es

MANUEL DE LEÓN

Instituto de Matemáticas y Física Fundamental, CSIC, Serrano 123, 28006 Madrid, Spain. e-mail: mdeleon@imaff.cfmac.csic.es 